\title{
RELATIVE PREDICATIVITY AND DEPENDENT RECURSION IN SECOND-ORDER SET THEORY AND HIGHER-ORDER THEORIES
}

\author{
SATO KENTARO
}

\begin{abstract}
This article reports that some robustness of the notions of predicativity and of autonomous progression is broken down if as the given infinite total entity we choose some mathematical entities other than the traditional $\omega$. Namely, the equivalence between normal transfinite recursion scheme and new dependent transfinite recursion scheme, which does hold in the context of subsystems of second order number theory, does not hold in the contexts of subsystems of second order set theory where the universe $V$ of sets is treated as the given totality (nor in the context of those of $n+3$-th order number or set theories, where the class of all $n+2$-th order objects is treated as the given totality).
\end{abstract}

§1. Introduction. Predicativism is a mathematical standpoint which could be said to be between Platonism and Constructivism, and whose origin goes back to Poincaré and Russell. While natural numbers are accepted as a totality, other infinite entities are not and so-called "vicious circles", those definitions which depend on the totality of the class the defined sets belong to, are rejected. Thus, traditionally, $\omega$ is the only infinite entity whose totality is accepted. However, Feferman [3, p.617] stated that the predicativity is a relative notion, and we can consider other kinds of predicativity, relative to various structures. Among them, Feferman mentioned "predicativity given the notion of the cumulative hierarchy of sets" as an example. This seems to be an extreme case because the totality of it might contradict the standard view of open-endedness. We can however consider also predicativity given (the totality of) $\mathcal{P}(\omega)$ (i.e., real numbers) and predicativity given $\mathcal{P}(\mathcal{P}(\omega)$ ) (or equivalently, the class of all functions).

What kinds of mathematical discussion can be justified from these predicative standpoints? There have been so many arguments for the traditional one:

(A) Feferman [3, p.605] took as the limit of predicativity (in the traditional sense) the closure under autonomous progression of ramified hierarchy, each level $R_{\alpha}$ of which consists of all those sets (of natural numbers) definable by quantifiers over lower levels (in modern terms, $R_{\alpha+1}=\mathcal{P}(\omega) \cap \operatorname{Def}\left(R_{\alpha}\right)$ ). Thus the class of all sets of natural numbers is not given as a totality, but always being generated. Since all the (meaningful) formulae must be formalized in the ramified way, the quantifiers varying over the whole ramified hierarchy make no sense.

(B) Since the ramified hierarchy can be simulated by iterated elementary comprehension by the use of universal formula, and since the latter is defined in the 
former, autonomous progression of ramified hierarchy could be identified with the autonomous progression of iterated elementary comprehension:

since the totality of $\omega$ is accepted, number quantifiers make sense and so elementary comprehension should be accepted; once it is accepted, iterated application of it along a primitive recursive ordinal $\alpha$ should be accepted, provided a well-orderedness proof of $\alpha$ is accepted.

As the aforementioned constraint in (A), the schematic axiom (i.e., induction) for the formulae containing second order quantifiers not bound by levels of the ramified hierarchy should not a priori be accepted. Since those quantifiers with such bounds are coded by first order quantifiers (for detail, see $\S 5$, especially before Definition 17 ), the schemata are restricted to elementary ones ${ }^{1}$.

(C) One can argue that the well-orders are not necessarily coded by primitive recursive relations, but can be any elementary formulae with free variables, provided that the well-orderedness is proved universally (see Footnote 6 for detail).

(D) One can further argue that the rule "..., provided a well-orderedness proof is accepted" can be replaced by an implication "..., if it is a well-order". The resulting axiom should be called internalized autonomous progression of elementary comprehension. In the literature (e.g., [15]), it is called transfinite recursion.

We are not discussing which is right here. Whichever we choose, we can say:

if we have the well-orderedness of $\alpha_{1}$ by means of iterated (elementary) comprehension along $\omega$, and if we have the well-orderedness of $\alpha_{2}$ by means of iterated comprehension along $\alpha_{1}, \ldots$, then the iterated comprehension along $\alpha_{n}$ should be accepted (for standard $n$ ). ${ }^{2}$

However, the natural question arises: even if we agree that predicativity should posses some of these closure properties, why is it sufficient? Is there another type of progression that should be accepted predicatively? Particularly,

(E) Once having accepted (internalized) autonomous progression, should not we accept also autonomous progression of autonomous progression itself? Namely,

If the well-orderedness of $\alpha$ and of $\beta_{0}$ has been accepted and if, only by already accepted reasonings, we have a derivation, uniformly in $\xi \in \alpha$, from iterated comprehension along $\sum_{\eta<\xi} \beta_{\eta}$ to the well-orderedness of $\beta_{\xi}$, then iterated comprehension along $\sum_{\xi \in \alpha} \beta_{\xi}$ should be accepted.

Why is this called autonomous progression of autonomous progression? In one step of autonomous progression (of iterated elementary comprehension) mentioned above, the progression from $\alpha_{i}$ to $\alpha_{i+1}$ is given by the elementary comprehension iterated along the given well-order $\alpha_{i}$. Now the one step progression from $\alpha$ to $\sum_{\xi \in \alpha} \beta_{\xi}$ is given by the simple autonomous progression (from $\sum_{\eta<\xi} \beta_{\eta}$ to $\beta_{\xi}$ ) iterated along the given well-order $\alpha$.

(F) There seems to be no reason to stop at (E) "2-fold autonomous progression". We should also accept 3 -fold one (namely, autonomous progression of (E)), 4-fold one, and so on.

\footnotetext{
${ }^{1}$ This restriction does not affect the proof-theoretic strength, but it does with the following relaxations.

${ }^{2}$ Moreover, it was shown that, by this process, the limit of such $\alpha_{n}$ 's is Feferman-Schütte ordinal $\Gamma_{0}$, whichever from (A)-(D) (actually, from all (A)-(F') defined below) we choose.
} 
(B')-(F') Or, "elementary" in (B)-(F) could be replaced by $\Delta_{e}^{1}$, (i.e., essentially $\left.\Delta_{1}^{1}\right)$, since it is "recognizable invariance" [3, p.606] during the generating process.

Nevertheless, the question which of $(\mathrm{A})-(\mathrm{F})$ (or $\left.-\left(\mathrm{F}^{\prime}\right)\right)$ is the right one does not affect the limit of the fragment of mathematics justifiable from the traditional predicativity. For, we can prove the proof-theoretic equivalence (or equiconsistency) between the system associated with the apparently strongest, namely the internalized version of $\left(\mathrm{F}^{\prime}\right)$, and that with $(\mathrm{A})$, if we employ plausible formulations as we will below (and, moreover, internalized versions of $(\mathrm{D}),(\mathrm{E}),(\mathrm{F})$, (D'), (E') and (F') are all logically equivalent, not only proof-theoretically).

This is the robustness that we will show to break down in the contexts of the other kinds of predicativity listed at the beginning. It seems possible to claim that this robustness is a special feature of $\omega$, and that, in the general case, it breaks down. We will see other special features of $\omega$, as byproducts of the proof.

More precisely, the contents of the present article are as follows.

First, we are working in the frameworks of (a) second order set theory, whose language is $\mathcal{L}_{S}^{2}$ and of (b) $n+2$-th order number (and set) theory, whose language is $\mathcal{L}_{N}^{n+2}$ (and $\mathcal{L}_{S}^{n+2}$ ) for $n \geq 1$. As base theories, in each framework we employ

(a) Neumann-Bernays-Gödel set theory (or NBG for short), which contains:

- Zermelo-Fraenkel set theory (with and without choice) with separation and replacement schemata applied to elementary $\mathcal{L}_{S}^{2}$-formulae,

- the comprehension schema for elementary $\mathcal{L}_{S}^{2}$-formulae,

for the requirement that the universe $V$ of sets is a given totality; and,

(b) so-called the Bernays-Gödel extension (or, sometimes, predicative extension) of full $n+1$-th order number (and set) theory, which contains:

- all the axioms of full $n+1$-th order number (or set) theory with all the schemata applied to any $n+1$-th order $\mathcal{L}^{n+2}$-formulae,

- comprehension schema (yielding subclasses of the given totality, namely $n+2$-th order objects) for all $n+1$-th order $\mathcal{L}^{n+2}$-formulae,

for the requirement that $n+1$-th and lower order objects are all given (where " $n+1$-th order" means containing no $n+2$-th order quantifiers).

Note that if we allow $n=0$ then (b) includes both (a) and the framework of second order number theory with the base theory $\mathbf{A} \mathbf{C A} \mathbf{A}_{0}$, as special cases.

In these frameworks, we define: the transfinite recursion scheme $\Delta_{0}^{n+1} \mathbf{- T R}$, which allows iteration of $\Delta_{0}^{n+1}$-comprehension (i.e., elementary comprehension in (a); and $n+1$-th order comprehension in (b)) along any well-order whose domain is (included in) the given totality, as a formalization of "single-fold" internalized autonomous progression of the comprehension; and a dependent transfinite recursion scheme $\Delta_{0}^{n+1}-\mathbf{T} \mathbf{R}^{\sharp}$ as a formalization of the simplest non-trivial instance of multi-fold internalized autonomous progression; as well as those for $\Delta_{e}^{n+1}$. (Note that $\Delta_{0}^{1}$-TR in $\mathcal{L}_{N}^{2}$ is called ATR.) We will see that any of $\Pi_{1}^{n+1}$ reduction and $\Delta_{0}^{n+1}$ positive fixed point axioms, which both have been known to be predicatively justifiable in $\mathcal{L}_{N}^{2}$, imply $\Delta_{0}^{n+1}-\mathbf{T R}^{\sharp}$.

As our main theorem, we prove that $\Delta_{0}^{n+1}-\mathbf{T R} \sharp$ (actually the "external" version of it) implies the consistency of $\Delta_{0}^{n+1}$-TR (without $\sharp$ ) with the base theories. (Since "internalized" $\Delta_{0}^{n+1}-\mathbf{T R}$ obviously implies the "external" counterpart, the separation between single- and multi-fold ones is now established for 
both internal and external versions.) We also see the underivability of $\Delta_{1}^{n+1}$ comprehension from $\Delta_{0}^{n+1} \mathbf{- T R}$ (without $\sharp$ ). These proofs are uniform for (a) and (b), whereas $\Delta_{e}^{1}$ - $\mathbf{T} \mathbf{R}^{\sharp}, \Delta_{e}^{1}$-TR, $\Delta_{0}^{1}-\mathbf{T} \mathbf{R}^{\sharp}$ and $\Delta_{0}^{1}$ - $\mathbf{T R}$ are all equivalent (so imply $\Delta_{1}^{1}$-comprehension) in $\mathcal{L}_{N}^{2}$. The basis of this difference will also be discussed.

Thus the relations among the central notions in the traditional predicativity heavily depend on the special feature of $\omega$. We conclude that relative predicativity requires more studies than the trivial analogy to the traditional one.

§2. Definitions of formal systems. Though we will obtain the results in both (a) second-order set theory, and (b) higher order number and set theories, we will work in one language with one base theory in the actual technicality. In this section, we give formal definitions of the languages $\mathcal{L}_{N}^{n+2}$ and $\mathcal{L}_{S}^{n+2}$ and base theory $\Delta_{0}^{n+1}-\mathbf{C A}_{0}$, explain the way to treat (a) and (b) uniformly, and, in $\S 4$, define in this way several axiom schemata, to be added to the base theory.

Definition 1. (i) The language $\mathcal{L}_{S}^{2}$ of second order set theory (or $\mathcal{L}_{N}^{2}$ of second order number theory) is two-sorted one, which contains the language of first order set theory (or first order number theory, respectively) as the fragment of the first sort, and which has a relation symbol $\in$ between the two sorts.

(ii) For $n \geq 1$, the languages $\mathcal{L}_{N}^{n+2}$ and $\mathcal{L}_{S}^{n+2}$ of $n+2$-th order number and set theories are $n+2$-sorted ones, which contain $\mathcal{L}_{N}^{2}$ and $\mathcal{L}_{S}^{2}$, respectively, as the fragments of the first two sorts, and which have equalities $={ }^{k}$ for $k+1$-th order for $k<n+1$ and relation symbols $\in^{k}$ between $k$-th and $k+1$-th sorts for any $k$.

Equality is not primitive for the highest order, but is defined by extensionality.

Here $k+1$-th order objects are intended to represent sets of $k$-th order ones. We omit the subscripts ' $N$ ' and ' $S$ ' when it is clear from the context or not important. When we need to know the orders of variables, we shall provide superscripts to variables. The superscript $k$ in $\epsilon^{k}$ is omitted when it is clear.

In what follows, we assume $n \geq 0$ and treat $\mathcal{L}_{N}^{n+2}$ and $\mathcal{L}_{S}^{n+2}$ uniformly, by the convention: upper-case Latin letters denote $n+2$-th order objects, and lower-case ones without superscripts denote those of the lower (i.e., $\leq n+1$-th) orders.

Definition 2. For $k \leq n$, an $\mathcal{L}^{n+2}$-formula is said to be $k+1$-th order (or elementary if $k=0$ ) or $\Delta_{0}^{k+1}$, if it contains no $k+2$-th nor higher order quantifiers (but it may contain $k+2$-th and higher order parameters). An elementary formula is called $\Delta_{0}^{0}$ if it contains no unbounded quantifiers.

For $k \leq n+1$, an $\mathcal{L}^{n+2}$-formula is called $\Pi_{1}^{k}$ or $\Sigma_{1}^{k}$ if it is of the form $\left(\forall x^{k}\right) \psi(x)$ or $\left(\exists x^{k}\right) \psi(x)$ respectively, where $\psi$ is $\Delta_{0}^{k}$ and $x^{k}$ is of $k+1$-th order. An $\Delta_{0}^{k+1}$ formula $\varphi$ is $\Pi_{e}^{k}$ (essentially $\Pi_{1}^{k}$ ) if all $k+1$-th order quantifiers in $\varphi$ are either positive universal or negative existential, and is $\Sigma_{e}^{k}$ if $\neg \varphi$ is $\Pi_{e}^{k}$.

For a class $\Gamma$ of formulae, the relativizations, e.g., $\Pi_{k}^{m}(\Gamma)$, are defined as usual.

Here we can find some conflict between the two counting systems: " $n+1$-th order" objects are also called "type $n$ " objects. The term " $n$-th order number theory" and $\mathcal{L}^{n+2}$ are from the former, and $\Sigma_{j}^{k}$ 's and $x^{k}$ are from the latter. Since both have been firmly standard, we have to get along with this conflict.

The most important feature of $\mathcal{L}^{n+2}$ is the ability to code pairs of lower order, by which we have the usual contraction rules on quantifiers. 
Definition 3. (i) The first order (or type-0) pairing $\langle x, y\rangle^{0}$ denotes Gödel's pairing $(x+y)(x+y+1) / 2+y$ in $\mathcal{L}_{N}^{n+2}$, and Mostowski pairing $\{\{x, y\},\{x\}\}$, in $\mathcal{L}_{S}^{n+2}$, of first order $x$ and $y$.

(ii) The $k+2$-th order (or type- $k+1$ ) pairing is defined (with Extensionality) by $u \in^{k+1}\langle x, y\rangle^{k+1} \leftrightarrow\left(\exists v^{k} \in^{k+1} x\right)\left(u=\langle 0, w\rangle^{k}\right) \vee\left(\exists w^{k} \in^{k+1} y\right)\left(u=\langle 1, w\rangle^{k}\right)$,

where 0 and 1 are fixed distinct $k+1$-th order objects.

(iii) For $k+1$-th order $u$ and $k$-th order $z,(u)_{z}$ denotes $\left\{x^{k-1} \mid\langle z, x\rangle^{k-1} \in u\right\}$.

(iv) Similarly, for $n+1$-th order $y,(X)_{y}$ denotes the "class" $\left\{z^{n} \mid\langle y, z\rangle^{n} \in X\right\}$.

Here "class" means a collection of those objects satisfying a fixed formula (or what is called an abstract). The use of this term might cause a confusion, since in the context of second order set theory, it also refers to "objects of second order". In the present article, however, we never make the use of the latter kind.

For formulae $\varphi(X)$ and $\psi(x), \varphi\left(\left\{x^{n} \mid \psi(x)\right\}\right)$ denotes the result of replacing all those subformulae of the form $t \in X$ by $\psi(t)$ in $\varphi(X)$.

Definition 4. $\mathrm{WF}(W)$ is defined as $(\forall Y) \mathrm{TI}[Y](W)$, where

$$
\mathrm{TI}[Y](W) \equiv\left(\forall x^{n}\right)\left[\left(\forall y^{n} \in(W)_{x}\right)(y \in Y) \rightarrow x \in Y\right] \rightarrow\left(\forall x^{n}\right)(x \in Y) .
$$

This expresses the well-foundedness of an $n+2$-th order relation $W$. This is a priori $\Pi_{1}^{n+1}$, and the question if it is equivalently $\Delta_{0}^{n+1}$ will be crucial.

Definition 5. $\Delta_{0}^{n+1}-\mathbf{C A}_{0}$ denotes the $\mathcal{L}_{N}^{n+2}$ - or $\mathcal{L}_{S}^{n+2}$-theory, consisting of

(0) Extensionality for lower order: for $k<n$

$$
\left(\forall x^{k+1}, y^{k+1}\right)\left[\left(\forall u^{k}\right)\left(u \in \in^{k+1} x \leftrightarrow u \in \in^{k+1} y\right) \rightarrow x={ }^{k+1} y\right]
$$

(1) $\Delta_{0}^{n+1}$-CA: $(\exists Z)\left(\forall z^{n}\right)(z \in Z \leftrightarrow \varphi(z))$ for a $\Delta_{0}^{n+1}$-formula $\varphi$ free from $Z$;

(2) $k+2$-th order $\Delta_{0}^{n+1}$ comprehension: for $k<n$ : $\left(\exists u^{k+1}\right)\left(\forall y^{k}\right)\left(y \in^{k+1} u \leftrightarrow \varphi(y)\right)$ for any $\Delta_{0}^{n+1}$-formula $\varphi$ free from $u$

(3) the global well-order among $n+1$-th order objects: $(\exists W)\left[\mathrm{WF}(W) \wedge\left(\forall x^{n}, y^{n}\right)\left(\langle x, y\rangle^{n} \in W \vee x=y \vee\langle y, x\rangle^{n} \in W\right)\right]$

(4) axioms for the first order part: $\left(\right.$ in $\mathcal{L}_{N}^{n+2}$ )

- the axiom of discrete-ordered semi-ring;

- $\left[\varphi(0) \wedge\left(\forall s^{0}\right)(\varphi(s) \rightarrow \varphi(s+1))\right] \rightarrow\left(\forall s^{0}\right) \varphi(s)$, for any $\Delta_{0}^{n+1}$-formula $\varphi$; $\left(\right.$ in $\mathcal{L}_{S}^{n+2}$ )

- the axioms of extensionality, empty set, pair, union, power set, infinity;

- $\left.\left(\forall u^{0}\right)\left[\left(\forall v^{0} \in u\right) \varphi(v) \rightarrow \varphi(u)\right)\right] \rightarrow\left(\forall u^{0}\right) \varphi(u)$, for any $\Delta_{0}^{n+1}$-formula $\varphi$;

- $\left(\forall u^{0} \in y^{0}\right)\left(\exists ! v^{0}\right) \varphi(u, v, y) \rightarrow\left(\exists z^{0}\right)\left(\forall u^{0} \in y\right)\left(\exists v^{0} \in z\right) \varphi(u, v, y)$ for any $\Delta_{0}^{n+1}$ formula $\varphi$ free from $z$ and

- $\left(\exists z^{0}\right)\left(\forall u^{0}\right)[u \in z \leftrightarrow u \in y \wedge \varphi(u, y)]$, for any $\Delta_{0}^{n+1}$-formula $\varphi$ free from $z$.

REMARK 6. $\Delta_{0}^{1}-\mathbf{C A}_{0}$ is $\mathbf{A} \mathbf{C A}_{0}$ in $\mathcal{L}_{N}^{2}$ and is Neumann-Bernays-Gödel set theory NBG in $\mathcal{L}_{S}^{2}$, which are known to be conservative over Peano arithmetic PA and Zermelo-Fraenkel set theory with choice $\mathbf{Z F C}$ respectively. More generally, $\Delta_{0}^{n+1}-\mathbf{C A}_{0}$ we have defined is what is known as the Bernays-Gödel extension of (so, conservative over) the full $n+1$-th order number or set theory.

The restriction in our base theory of all the schematic axioms to $\Delta_{0}^{n+1}$, i.e., the formulae without $n+2$-th order quantifiers can be explained as follows: since 
we accepted the totality of the class of all $n+1$-th order objects, the formulae containing only $n+1$-th and lower order quantifiers are meaningful, whereas those containing $n+2$-th order quantifiers are undermined, as discussed in (B) of $\S 1$.

REMARK 7. The terminology ' $\Delta_{0}^{n+1}-\mathbf{C A}_{0}$ ' comes from the fact that the "characteristic axiom" is $\Delta_{0}^{n+1} \mathbf{- C A}$. Actually, this is the only "essentially non- $\Delta_{0}^{n+1}$ " axiom: Axioms (0), (2) and (4) are (the universal closures of) $\Delta_{0}^{n+1}$-formulae, and, with a new constant for the global well-order, Axiom (3) can also be.

REMARK 8. By the canonical injection from $k+1$-th order part $(k \leq n)$ into $n+1$-th order part, defined as iterated singleton $x \mapsto\{x\}^{n-k}$, we have global well-orders $w^{k+1}=\left\{\langle x, y\rangle^{k} \mid\left\langle\{x\}^{n-k},\{y\}^{n-k}\right\rangle^{n} \in W\right\}$ among the $k+1$-th order.

§3. Global well-ordering and normal form theorem. It might seem strange that the higher order number and set theories contain Axiom (3). While the former has been investigated for a long time, it is unclear if the axiom is included in the standard formulation, since those of consistency strength above full second order number theory $\mathbf{Z}_{2}$ (but below $\mathbf{Z}_{n}$ for a fixed $n$ ) have not been considered so much (with few exceptions, e.g., Friedman's [6] famous result on determinacy, which deals with the higher order number theory in a variant of Gödel's constructible hierarchy). Though it could be claimed that the axiom is directly justified by our notion of "set of set of ... numbers", the author is not confident and, rather, would like to argue against it.

REMARK 9. However, from the viewpoint of proof-theoretic (or consistency) strength, this does not matter: The author is preparing a work [14] which establishes the equiconsistency between $\Delta_{0}^{n+1}-\mathbf{C A}_{0}$ (augmented by the additional axioms treated in the present article) with and without the axiom (3).

Moreover, for $n=0$, we will not use (3): in $\mathcal{L}_{N}^{2}$ it is redundant because of the usual order $<$ on $\omega$, and in $\mathcal{L}_{S}^{2}$ the replacement scheme (or, more precisely, the reflection principle) can substitute (3) in our discussion, as shown in $\S 8$.

Our first essential use of this axiom is in the proof of normal form theorem.

Proposition 10. Let $k \leq n+1$. Any $\Sigma_{1}^{k}$-formula is equivalent, over $\Delta_{0}^{n+1}-\mathbf{C A}_{0}$, to one in the following form, where $f: \mathcal{P}^{k} \rightarrow \mathcal{P}^{k}$ denotes $\left(\forall y^{k}\right)\left(\exists ! z^{k}\right)\left(\langle y, z\rangle^{k} \in f\right)$ and where $Q_{i}$ 's are alternating $\left(Q_{i} \equiv \exists\right.$ if $k-i$ is even, and $Q_{i} \equiv \forall$ otherwise):

$$
\underbrace{\left(\exists f^{k}: \mathcal{P}^{k-1} \rightarrow \mathcal{P}^{k-1}\right)}_{k+1 \text {-th order q.f. }} \cdots \underbrace{\left(Q_{1} h^{1}: \mathcal{P}^{0} \rightarrow \mathcal{P}^{0}\right)}_{\text {2nd }} \underbrace{\left(Q_{0} z^{0}\right)}_{\text {1st }} \underbrace{\varphi(f ; g ; \cdots ; h ; z)}_{\Delta_{0}^{0} \text { part }}
$$

Proof. By Remark 8, we have the axiom of choice for all orders except the highest: for $k<n$ and for any $\Delta_{0}^{n+1}$-formula $\varphi$,

$\Delta_{0}^{n+1}-\mathbf{C A}_{0} \vdash(\forall \vec{x}, \vec{X})\left[\left(\forall y^{k}\right)\left(\exists u^{k+1}\right) \varphi(y, u, \vec{x}, \vec{X}) \leftrightarrow\left(\exists u^{k+1}\right)\left(\forall y^{k}\right) \varphi\left(y,(u)_{y}, \vec{x}, \vec{X}\right)\right]$.

By this and dual, any $\Sigma_{1}^{k}$ - formula is equivalent to one in the form:

(*)

$$
\left(\exists y^{k}\right) \underbrace{\left(Q_{1} x_{1}^{k-1}\right) \cdots\left(Q_{k} x_{m}^{k-1}\right)}_{k \text {-th order q.f. }} \cdots \underbrace{\left(Q_{1} z_{1}^{0}\right) \cdots\left(Q_{l} z_{l}^{0}\right)}_{\text {1st order q.f. }} \underbrace{\varphi\left(y ; x_{1}, \cdots, x_{m} ; \cdots ; z_{1}, \cdots, z_{l}\right)}_{\Delta_{0}^{0} \text { part }} .
$$


The well-orders also allow us to use the method of Skolem function on each order $\leq n+1$. By generalizing the proof in second order number theory (see [15, Lemma V.1.4]), we have: for any $j \leq n$ and $\Sigma_{1}^{j+1}$-formula $\varphi(\vec{x}, \vec{X})$,

$$
\Delta_{0}^{n+1}-\mathbf{C A}_{0} \vdash(\forall \vec{x}, \vec{X})\left[\varphi(\vec{x}, \vec{X}) \leftrightarrow\left(\exists f^{j+1}: \mathcal{P}^{j} \rightarrow \mathcal{P}^{j}\right)\left(\forall y^{j}\right) \psi(f, y, \vec{x}, \vec{X})\right]
$$

holds for some $\Delta_{0}^{j}$-formula $\psi\left(f^{j+1}, y^{j}, \vec{x}, \vec{X}\right)$. By taking the negation, we have a similar result for $\Pi_{1}^{j+1}$-formula. By applying these results to $(\star)$ from outside, we have the desired result.

DeFinition 11. Let $\sigma$ and $\pi$ be universal $\Sigma_{1^{-}}^{0}$ and $\Pi_{1}^{0}$-formulae respectively. $\Upsilon_{1}^{k}(c, \vec{x}, \vec{X}) \equiv\left(\exists f^{k}: \mathcal{P}^{k-1} \rightarrow \mathcal{P}^{k-1}\right) \cdots\left(Q_{1} h^{1}: \mathcal{P}^{0} \rightarrow \mathcal{P}^{0}\right) v\left(c, f^{k} ; \cdots ; h^{1} ; \vec{x}, \vec{X}\right)$ where $Q_{i}$ 's are alternating and where $v \equiv \pi$ for odd $k ; v \equiv \sigma$ otherwise.

Corollary 12. Let $k \leq n+1$. For any $\Sigma_{1}^{k}$-formula $\varphi(\vec{x}, \vec{X})$, there is $\ulcorner\varphi\urcorner \in \omega$ such that

$$
\Delta_{0}^{n+1}-\mathbf{C A}_{0} \vdash(\forall \vec{x}, \vec{X})\left[\Upsilon_{1}^{k}(\ulcorner\varphi\urcorner, \vec{x}, \vec{X}) \leftrightarrow \varphi(\vec{x}, \vec{X})\right] .
$$

§4. Additional axiom schemata. Following the convention on the distinction of upper and lower cases, we can define several axiom schemata uniformly.

Definition 13. For a class $\Gamma$ of formulae, define the following axiom schemata:

$\Gamma$-CA: $(\exists Z)\left(\forall z^{n}\right)(z \in Z \leftrightarrow \varphi(z))$,

$\Delta(\Gamma)$-CA: $\left(\forall z^{n}\right)(\varphi(z) \leftrightarrow \neg \psi(z)) \rightarrow\{\varphi\}-\mathbf{C A}$,

$\Gamma$-FP: $(\exists Z)\left(\forall z^{n}\right)(z \in Z \leftrightarrow \varphi(z, Z))$ if $Y$ occurs only positively in $\varphi(z, Y)$,

$\Gamma$-Red: ${ }^{3}\left(\forall z^{n}\right)(\varphi(z) \vee \psi(z)) \rightarrow(\exists Z)\left(\forall z^{n}\right)[(z \in Z \rightarrow \varphi(z)) \wedge(z \notin Z \rightarrow \psi(z))]$,

$\Gamma$-Coll: $\left(\forall z^{n}\right)(\exists Y) \varphi(z, Y) \rightarrow(\exists Z)\left(\forall z^{n}\right)\left(\exists y^{n}\right) \varphi\left(z,(Z)_{y}\right)$

for $\Gamma$ formulae $\varphi(z, Y)$ and $\psi(z, Y)$ in which $Z$ does not occur (but parameters might). $\Delta_{\bullet}^{m}$ stands for $\Delta\left(\Pi_{\bullet}^{m}\right)$ which we will treat as if it were a class of formulae.

Definition 14. ${ }^{4}\left(\Delta_{0}^{n+1}\right)^{-}$is the class of all those $\Delta_{0}^{n+1}$-formulae that have no $n+2$-th order parameters. $\widehat{I D}_{1}^{n+1}$ is the system $\Delta_{0}^{n+1}-\mathbf{C A}_{0}+\left(\Delta_{0}^{n+1}\right)^{-} \mathbf{- F P}$.

The proof of (ii) below is literally the same, by the virtue of our abbreviations, as that in the second order case, e.g., in [7, Proposition 4.5].

Lemma 15. Over $\Delta_{0}^{n+1}-\mathbf{C A}_{0}$, (i) $\Gamma$-CA implies $\Gamma$-Red, (ii) $\Sigma_{1}^{n+1}$-Coll implies $\Sigma_{1}^{n+1}$-Red and (iii) $\Gamma$-Red implies $\Delta(\Gamma)$-CA.

Proof. Since (i) and (iii) are trivial, we prove (ii) by working in $\Delta_{0}^{n+1}-\mathbf{C A} \mathbf{A}_{0}$. Let $\varphi$ and $\psi$ be $\Delta_{0}^{n+1}$ and $(\forall z)[(\exists X) \varphi(z, X) \vee(\exists X) \psi(z, X))$, i.e., $(\forall z)(\exists Y)[\varphi(z, Y) \vee$ $\psi(z, Y)]$. By $\Sigma_{1}^{n+1}$-Coll, $(\forall z)(\exists y)\left[\varphi\left(z,(Z)_{y}\right) \vee \psi\left(z,(Z)_{y}\right)\right]$ for some $Z$. Now,

$$
(\exists y) \varphi\left(z,(Z)_{y}\right) \rightarrow(\exists X) \varphi(z, X) \text { and } \neg(\exists y) \varphi\left(z,(Z)_{y}\right) \rightarrow(\exists X) \psi(z, X) .
$$

Thus $X=\left\{z \mid(\exists y) \varphi\left(z,(Z)_{y}\right)\right\}$, yielded by $\Delta_{0}^{n+1} \mathbf{- C A}$, is what is required.

\footnotetext{
${ }^{3}$ This is equivalent, in classical logic, to what is called $(\neg \Gamma)$-Sep in Simpson's book [15]. Since the term "separation" is confusing in the present context, we use "reduction" instead.

${ }^{4}$ Strictly $\widehat{I D}_{1}^{n+1}$ should be formulated in an extension of $\mathcal{L}^{n+1}$ with predicates, rather than $\mathcal{L}^{n+2}$. If we identify the predicates with the $n+2$-th order objects required in the scheme $\left(\Delta_{0}^{n+1}\right)^{-}-\mathbf{F P}$, the two formulations are equivalent.
} 
$\S 5$. Formalizing (internalized) autonomous progression. We give some formalizations of autonomous progression, and discuss them in our new setting. First recall the standard formulation of iterated comprehension.

Definition 16. Assume $\mathrm{WF}(W)$. $H$ is said to code the iterated comprehension with a formula $\varphi$ along $W$, if $\operatorname{Hier}[\varphi](H, W)$ holds where

$$
\operatorname{Hier}[\varphi](H, W) \equiv\left(\forall w^{n}\right)\left[(H)_{w}=\left\{y^{n} \mid \varphi\left(w, y,(H)_{W x}, W\right)\right\}\right],
$$

and where $(H)_{W x}$ denotes $\left\{\left\langle w^{\prime}, z\right\rangle^{n} \in H \mid w^{\prime} \in(W)_{w}\right\}$.

We see briefly how the iterated comprehension simulate the ramified hierarchies relative to $\mathcal{P}(\omega), \mathcal{P}^{2}(\omega), V$ etc., in the same way as that relative to $\omega$.

Assume $\operatorname{Hier}[\Phi](H, W)$, where $\Phi(w, y, Y, W ; \vec{x}, \vec{X})$ is the following $\Sigma_{2}^{n}$-formula:

$\left(\exists z^{n}\right)(\exists c \in \omega)\left[y=\langle c, z\rangle^{n} \wedge\left(" w\right.\right.$ is limit in $\left.W^{\prime} \rightarrow \Upsilon_{1}^{n}(c, z, Y ; \vec{x}, \vec{X})\right) \wedge$

$\left(\exists w^{\prime n}\right)\left(" w\right.$ is succ. of $w^{\prime}$ in $\left.\left.W^{\prime \prime} \rightarrow\left(\exists u^{n}\right)\left(\forall v^{n}\right)\left(\left\langle u,\langle v, z\rangle^{n}\right\rangle^{n} \in\left((Y)_{w^{\prime}}\right)_{c}\right)\right)\right]$.

Here $c \in \omega$ is regarded, via the canonical injection, as an $n+1$-th order object.

Assume for convenience that $W$ is a linear order. If $w$ is a limit in $W$,

$$
\left.(H)_{w}=\left\{y \mid \Phi\left(w, y,(H)_{W w}, W ; \vec{x}, \vec{X}\right)\right\}=\left\{\langle c, z\rangle^{n} \mid \Upsilon_{1}^{n}\left(c, z,(H)_{W w} ; \vec{x}, \vec{X}\right)\right)\right\},
$$

and so $\left((H)_{w}\right)_{\ulcorner\varphi\urcorner}=\left\{z^{n} \mid \varphi\left(z,(H)_{W w} ; \vec{x}, \vec{X}\right)\right\}$. If $w^{+}$is the successor of $w$,

$$
(H)_{w^{+}}=\left\{\langle c, z\rangle^{n} \mid\left(\exists u^{n}\right)\left(\forall v^{n}\right)\left(\left\langle u,\langle v, z\rangle^{n}\right\rangle^{n} \in\left((H)_{w}\right)_{c}\right\},\right.
$$

and so $\left((H)_{w^{+}}\right)_{\ulcorner\varphi\urcorner}=\left\{z^{n} \mid\left(\exists u^{n}\right)\left(\forall v^{n}\right) \varphi\left(\left\langle u,\langle v, z\rangle^{n}\right\rangle^{n},(H)_{W w} ; \vec{x}, \vec{X}\right)\right\}$. Iterating this process, we can see that any $n+2$-th order object definable by a $\Sigma_{2 i+1^{-}}^{n}$ formula with parameters $(H)_{W w}, \vec{x}$ and $\vec{X}$ can be described by $\left((H)_{w^{+i}}\right)_{c}$ for some $c \in \omega$. Thus, if the next limit point $w^{+\omega}$ exists, all those $n+2$-th order objects $\Delta_{0}^{n+1}$-definable with the parameters are of the form $\left(\left((H)_{W w^{+\omega}}\right)_{x}\right)_{y}$.

This means that the first level of the ramified hierarchy relative to the parameters $(H)_{W w}, \vec{x}$ and $\vec{X}$ is exactly $\left\{\left(\left((H)_{W w^{+\omega}}\right)_{x}\right)_{y} \mid x, y \in \mathcal{P}^{n}\right\}$ (where $\mathcal{P}^{n}$ is the "class" of all $n+1$-th order objects) and that the quantifiers ranging over the first level of the ramified hierarchy can be coded by $n+1$-th order quantifiers. Thus

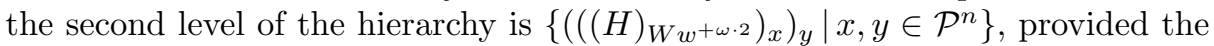
next limit $w^{+\omega \cdot 2}$ exists.

Therefore we can conclude that iterated $\Delta_{0}^{n+1}$-comprehension (i.e., comprehension for $n+1$-order formulae) can simulate the "ramified hierarchy given the totality of the class of all the $n+1$-th order objects", in such a way that $n+2$ th order quantifiers bounded by levels of the hierarchy are coded by $n+1$-order quantifiers. Conversely, $H$, required in $\operatorname{Hier}[\varphi](H, W)$, is in the level o.t. $(W)+1$ of the ramified hierarchy. ${ }^{5}$ Now we can safely move from (A) to (B).

The next question is how to formalize autonomous progression of iterated comprehension. The most naive way to do this seems to be $\Delta_{0}^{n+1} \mathbf{- T R}$, where

Definition 17. $\Gamma$-TR: $\mathrm{WF}(W) \rightarrow(\exists H) \operatorname{Hier}[\varphi](H, W)$,

$\Delta(\Gamma)$-TR: $(\forall w, y, Y)[\varphi(w, y, Y, W) \leftrightarrow \neg \psi(w, y, Y, W)] \rightarrow\{\varphi\}-\mathbf{T R}$,

for any $\Gamma$ formulae $\varphi$ and $\psi$ both with no occurrences of $H$.

\footnotetext{
${ }^{5}$ In the terminology introduced below, this discussion also shows that, over $\Delta_{0}^{n+1}-\mathbf{C A}_{0}$, $\Delta_{0}^{n+1} \mathbf{- T R}$ or $\mathbf{- T R R}$ is equivalent to $\Sigma_{2}^{n}$ - TR or - TRR. Note that " $w$ is limit in $W$ " is $\Pi_{2}^{n}$.
} 
However, here was a difficulty in $\mathcal{L}_{N}^{2}$ : the notion of well-foundedness (formalized as $\mathrm{WF}(W)$ ) is not predicative, since the class of all $\omega$-chains is not given as a totality. One idea to avoid this was to use the following rule with $\Gamma=\Delta_{0}^{n+1}$.

$\Gamma$-TRR: For any $\Gamma$-formulae $\varphi$ and $\psi$ (identified with $\left\{\langle x, y\rangle^{n} \mid \psi(x, y)\right\}$ ) free from $Y$ and $H$, from a proof of $\operatorname{TI}[Y](\psi)$, we can deduce $(\exists H) \operatorname{Hier}[\varphi](H, \psi)$.

Why was this claimed to avoid the difficulty? As explained in [3, p.605, 1l.9-26] in a slightly different formulation, if we have a proof of $\mathrm{TI}[Y](\psi)$ without any undischarged assumptions, we can substitute any formula into the undetermined variable $Y$, and so the well-foundedness holds at any (later) stages of generating process of sets. Now it seems reasonable to consider $\Delta_{0}^{n+1} \mathbf{- T R R}$ and $\Delta_{0}^{n+1} \mathbf{- T R}$ as the formalizations of (C) autonomous progression (because $\psi$ may contain free variables $)^{6}$, and of (D) internalized autonomous progression respectively. It is known that, in $\mathcal{L}_{N}^{2}$, these two are proof-theoretically equivalent.

However, the difficulty above does not seem to be a real difficulty in our setting (except $\mathcal{L}_{N}^{2}$ ), and seems to be special to the traditional predicativity. It is true that $\mathrm{WF}(W)$ is $\Pi_{1}^{n+1}$ and that, in general, $\Pi_{1}^{n+1}$-formulae are illegitimate from the predicative viewpoint. Nonetheless, the $\Pi_{1}^{n+1}$-ness of well-foundedness is only because of our choice of formulation. Actually, in our setting (except $\mathcal{L}_{N}^{2}$ ), the well-foundedness can be expressed in a $\Delta_{0}^{n+1}$ way as we will see in $\S 8$, and so it is legitimate in our context. ${ }^{7}$ Once it is legitimately accepted, there seems to be no reason that forces us to formalize autonomous progression in the roundabout sort of way by the rule, but it seems reasonable to formalize it in a simple implication $\Delta_{0}^{n+1} \mathbf{- T R}$, the same as internalized autonomous progression.

REMARK 18. Anyway "internalized" $\Delta_{0}^{n+1}-\mathbf{T R}$ is $\Pi_{1}^{n+1}$-conservative over "external" $\Delta_{0}^{n+1}$-TRR in general $\mathcal{L}^{n+2}$. To the one-sided sequent calculus, we add:

$$
\overline{\vdash \Gamma, \phi} \text { Axiom } \frac{\vdash \Gamma, \neg \operatorname{Hier}[\varphi](H, W)}{\vdash \Gamma, \neg \mathrm{WF}(W)} \mathrm{TR}
$$

where (the universal closure of) $\phi$ is an axiom of $\Delta_{0}^{n+1}-\mathbf{C A}_{0}$, and where the eigenvariable condition applies to TR: $H$ does not occur in the lower sequent. Since $\Delta_{0}^{n+1}$-CA follows from $\Delta_{0}^{n+1} \mathbf{- T R}$ and hence from the rule TR, $\phi$ in Axiom can be restricted to $\Delta_{0}^{n+1}$-formulae, by the reason mentioned in Remark 7 .

If we have a proof ending in a $\Delta_{0}^{n+1}$-formula with free variables, then, by the usual partial cut elimination method, we have a proof ending in the same formula, in which all the cut rules are immediate after the axiom or of the following form:

$$
\frac{\vdash \Gamma_{1}, \neg \operatorname{Hier}[\varphi](H, W)}{\vdash \Gamma_{1}, \neg \mathrm{WF}(W)} \text { TR } \frac{\vdash \Gamma_{2}, \mathrm{TI}[Y](W)}{\vdash \Gamma_{2}, \mathrm{WF}(W)} \forall \text { Cut }
$$

\footnotetext{
${ }^{6}$ Concerning (B) which requires orders to be primitive recursive, there seems to be no reason for such restriction, since any order expressed by a $\Delta_{0}^{n+1}$-formula (with free set variables) is predicatively legitimate. The justification for the use of a free set variable $Y$ in $\mathrm{TI}[Y](-)$ (in the standard formulation of autonomous progression) seems to apply also to this allowance of free variables in the formula defining the order.

7 One might argue that the $\Delta_{0}^{n+1}$-ness of well-foundedness in our setting is a coincidence and that the well-foundedness is a priori $\Pi_{1}^{n+1}$. However, as a matter of fact, the $\Pi_{1}^{n+1}$ formalization $(\forall Y) \mathrm{TI}[Y](W)$ of well-foundedness itself is questionable, as discussed in $\S 6$.
} 
Therefore, $\Gamma_{1}, \Gamma_{2}$ here must consist only of $\Delta_{0}^{n+1}$-formulae. Define $\psi(x, y, W)$ by

$$
\psi(x, y, W) \equiv(\langle x, y\rangle \in W) \wedge\left(\neg \vee \Gamma_{2}\right) .
$$

If $\vee \Gamma_{2}$ holds then $\psi$ represents the trivial well-founded relation $\emptyset$; and if $\vee \Gamma_{2}$ does not, it represents $W$. Thus we can replace it by the following derivation:

$$
\begin{aligned}
& \vdash \Gamma_{2}, \mathrm{TI}[Y](W) \\
& \vdots \Lambda_{1} \\
& \frac{\vdash \operatorname{TI}[Y](\psi)}{\vdash(\exists H) \operatorname{Hier}[\varphi](H, \psi)} \operatorname{TRR}
\end{aligned}
$$

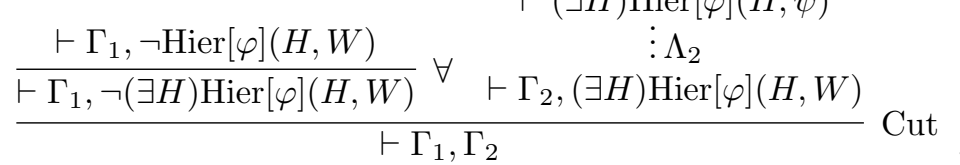

§6. Formalizing problem of WF. Following the referee's suggestion, we devote one section to the argument triggered by Footnote 7. As mentioned there, the fact the well-foundedness can be a legitimately expressible property in our predicative setting (except $\mathcal{L}_{N}^{2}$ ) leads us to a more fundamental question: if our formulation $(\forall Y) \mathrm{TI}[Y](W)$ of well-foundedness is appropriate or not.

The basic idea of Feferman's analysis of traditional predicativity could be summarized that the well-foundedness of an order guarantees the iterability of accepted operation along the order and that the taking ramified hierarchy is among such accepted operations to be iterated. It seems quite plausible that well-foundedness is a notion along which we can iterate operations. Nonetheless, why can we formalize it as $(\forall Y) \mathrm{TI}[Y](W)$, transfinite induction for set variables? Why does $(\forall Y) \mathrm{TI}[Y](W)$ guarantee the iterability of accepted operations?

It is true that, in the presence of full comprehension axiom, it guarantees that, because the property "we can iterate the operation up to $\alpha$ " of $\alpha$ can be substituted to the variable $Y{ }^{8}$ In our predicative standpoint, however, such a comprehension is not allowed and, particularly, the property "we can have the ramified hierarchy up to $\alpha$ " of $\alpha$ is not eligible to form a second order object, nor to be substituted to the variable $Y{ }^{9}$ For this reason, Feferman's argument might be said a "begging of question" from the impredicative standpoint.

This problem seems to be applied even to older principles called bar induction or bar recursion, whose origins go back to Brouwer. In modern terms, these principles are formulated as follows: if there is no infinite path through a tree, the transfinite induction or recursion along the reversed tree order is possible. In the presence of full comprehension, again, there is no problem, since we can use the property applied to induction or the property "recursion up to $t$ is possible" of a node $t$ to define an infinite path, assuming the induction or recursion fails. However, in the absence of relevant comprehension, it is not clear why the nonexistence of infinite path is enough for induction and recursion (except strange Brouwer's "proof", a confusion with the meta-level from the modern perspective). Again, this seems "question-begging" from the impredicative standpoint. ${ }^{10}$

\footnotetext{
${ }^{8}$ Thus, we no longer need to add the rule, since it is derivable from definitions and axioms.

${ }^{9}$ The question if this variable $Y$ is bounded by degree or not is irrelevant in this context.

${ }^{10}$ Intuitionistically, it seems to contain also "begging" from reductio ad absurdum.
} 
To overcome this problem, what is necessary is an argument justifying that $(\forall Y) \operatorname{TI}[Y](W)$ is the appropriate formalization of well-foundedness as the notion along which we can iterate accepted operations. Up to the author's best knowledge, there is no such an argument. Nevertheless, since the aim of the present paper is not criticism against Feferman's analysis of traditional predicativity but the analogous analysis for relative predicativity, we just claim that the formulation $(\forall Y) \mathrm{TI}[Y](W)$ is an approximation of the real well-foundedness, based on a partial help of the impredicative standpoint. Once we admit that this is nothing more than an approximation, one can ask: why do not we use an elementary approximation which would allow us to avoid the roundabout way by rule even for traditional predicativity? We simply do not know such a nice approximation, and the $\Pi_{1}^{1}$ completeness of well-foundedness in number theory implies that no such approximation can coincide with the real well-foundedness even on the impredicative standpoint. The author has to admit, however, that there is no guarantee that there is no better approximation of the notion. We use the formulation only because we (temporarily) resign ourselves to do so.

§7. Dependent transfinite recursion. Here we try to formalize (E) "autonomous progression of autonomous progression" (or "2-fold autonomous progression") from $\S 1$ in the simplest case, and obtain some basic results.

First, our formalization is the following.

Definition 19. For $k \in \omega, Y\left\lceil k\right.$ denotes $\left\{\left\langle\langle j, w\rangle^{n}, x\right\rangle^{n} \in Y \mid j<k\right\}$, and $\oplus_{k \in \omega} \prec_{Y}^{k}$ is defined by: $\langle j, w\rangle\left(\oplus_{k \in \omega} \prec_{Y}^{k}\right)\left\langle j^{\prime}, w^{\prime}\right\rangle \equiv j<j^{\prime} \vee\left(j=j^{\prime} \wedge w \prec_{Y}^{j} w^{\prime}\right)$.

$\Gamma$-TR $\mathbf{R}^{\sharp}:(\forall Y)(\forall k \in \omega) \mathrm{WF}\left(\prec_{Y \uparrow k}^{k}\right) \rightarrow(\exists H) \operatorname{Hier}[\varphi]\left(H, \oplus_{k \in \omega} \prec_{H\lceil k}^{k}\right)$,

$\Gamma$-TRR $\mathbf{T R}^{\sharp}$ : From a proof of TI $[Z]\left(\prec_{Y \nmid k}^{k}\right)$, we can infer $(\exists H) \operatorname{Hier}[\varphi]\left(H, \oplus_{k \in \omega} \prec_{H\lceil k}^{k}\right)$, both for any $\Gamma$-formula $\varphi(\langle k, w\rangle, y, Y, W ; \vec{x}, \vec{X})$ free from $H$ and for any $w \prec_{Y}^{k} w^{\prime}$ defined by a $\Gamma$-formula $\theta\left(w, w^{\prime}, k, Y ; \vec{x}, \vec{X}\right)$ free from $H$ and $Z$.

Analogously $\Delta(\Gamma)-\mathbf{T} \mathbf{R}^{\sharp}$ is defined (additionally with complementedness for $\theta$ ).

Here the well-founded relation $\oplus_{k \in \omega} \prec_{H \uparrow k}^{k}$ depends on the resulting $H$ in the following manner: since $H\left\lceil 0=\emptyset, \prec_{H \uparrow 0}^{0}\right.$ is fixed at first, not depending on $H$. Then, by (usual) transfinite recursion along $\prec_{H \uparrow 0}^{0},(H)_{\langle 0, x\rangle}$ 's are (thus $H\lceil 1$ is) determined, and so is $\prec_{H \uparrow 1}^{1}$. Then again, by usual recursion along it, $H\lceil 2$ is determined, and so on. This is why we call such a scheme dependent transfinite recursion. This argument shows "being unique if existing" below.

LEMMA 20. In the same syntactic situation of the previous definition,

$$
\Delta_{0}^{n+1}-\mathbf{C A}_{0} \vdash(\forall X \forall k \in \omega) \mathrm{WF}\left(\prec_{X\lceil k}^{k}\right) \rightarrow\left(\exists^{\leq 1} H\right) \operatorname{Hier}[\varphi]\left(H, \oplus_{k \in \omega} \prec_{H\lceil k}^{k}\right) .
$$

LEMMA 21. $\Gamma$-TR $+\Delta_{1}^{n+1}(\Gamma)-\mathbf{C A}+\Sigma_{1}^{n+1}(\Gamma)$-Ind implies $\Gamma$-TR $\mathbf{R}^{\sharp}$.

Proof. By the discussion before Lemma 20, $\Gamma$-TR $+\Sigma_{1}^{n+1}(\Gamma)$-Ind proves $(\exists ! H) \operatorname{Hier}[\varphi]\left(H, \oplus_{j \leq k} \prec_{H \backslash j}^{j}\right)$ for all $k$. Thus $\Delta_{1}^{n+1}(\Gamma)-\mathbf{C A}$ yields

$$
\left\{\left\langle\langle k, w\rangle^{n}, x\right\rangle^{n} \mid \exists H\left(\operatorname{Hier}[\varphi]\left(H, \oplus_{j \leq k} \prec_{H \nmid j}^{j}\right) \wedge\langle\langle k, w\rangle, x\rangle \in H\right)\right\},
$$

as a $n+2$-th order object, which is what we require for $\varphi \in \Gamma$ in $\Gamma$ - $\mathbf{T R}^{\sharp}$. 
Corollary 22. (a) In $\Delta_{0}^{n+1}-\mathbf{C A}_{0}+\Sigma_{1}^{n+1}(\Gamma)$-Ind, $\Delta_{1}^{n+1}(\Gamma)$-TR implies $\Gamma$-TR $\mathbf{T}^{\sharp}$ (b) Thus, $\Delta_{e}^{n+1} \mathbf{-} \mathbf{T R} \mathbf{R}^{\sharp}$ and $\Delta_{e}^{n+1} \mathbf{- T R}$ are equivalent over $\Delta_{0}^{n+1}-\mathbf{C A} \mathbf{A}_{0}+\Sigma_{e}^{n+1} \mathbf{- I n d}$.

Why can this be seen as the simplest non-trivial instance of (internalized) "autonomous progression of autonomous progression" (from §1) of comprehension? Let us try to formalize the following situation: $\prec^{k+1}$ is definable from $H_{k} ; H_{k}$ defined by transfinite recursion along $\prec^{k}$; and all these definitions are uniform 11 in $k \in \omega$. $\prec^{k}$ should be defined (uniformly in $k$ ) by a formula which may contain $H\left\lceil k\left(\approx H_{0} \oplus \cdots \oplus H_{k-1}\right)\right.$, and recursions along $\prec^{k}$ 's must be uniform, and so expressible by single Hier $[\varphi]$ along the sum $\prec^{0} \oplus \prec^{1} \oplus \cdots$.

This is the simplest among non-trivial ones, in the sense that the "preceding" order is restricted to $\omega$ (since if the "preceding" order is a standard number $n$ the statement is implied by non-dependent $\Gamma$-TR, as $n$-step autonomous progression). We will give a formalization of general 2 -fold one in $\S 10$.

Proposition 23. Over $\Delta_{0}^{n+1}-\mathbf{C A}_{0}, \Pi_{1}^{n+1}(\Gamma)$-Red implies $\Gamma$-TR $\mathbf{R}^{\sharp}$.

Proof. Following [15, Theorem V.5.1]. Let $\varphi$ be $\Gamma$, and define $\varphi_{+}$and $\varphi_{-}$:

$$
\varphi_{ \pm}(k, w, y) \equiv(\forall A)\left(\begin{array}{c}
\operatorname{Hier}[\varphi]\left(A,\left(\oplus_{j<k} \prec_{A\lceil j}^{j}\right) \oplus\left(\prec_{A\lceil k}^{k}\lceil w)\right)\right. \\
\rightarrow \pm \varphi\left(\langle k, w\rangle, y,(A)_{\left(\oplus_{j \leq k} \prec_{A\lceil j}^{j}\right)\langle k, w\rangle}, \vec{x}, \vec{X}\right)
\end{array}\right) .
$$

Intuitively, $\varphi_{ \pm}(k, w, y)$ assert that, for any attempt $A$ of the hierarchy up to $\langle k, w\rangle, \varphi\left(k, w, y, A^{k, w}\right)$ or $\neg \varphi\left(k, w, y, A^{k, w}\right)$, respectively, holds, where $A^{k, w}=$ $(A)_{\left(\oplus_{j \leq k} \prec_{A\lceil j}^{j}\right)\langle k, w\rangle}$. For fixed $k, w, y$, by Lemma 20 applied to $\prec_{Y}^{\prime j}$ defined below, such $A$ is unique if exists and so $(\forall k, w, y)\left(\varphi_{+}(k, w, y) \vee \varphi_{-}(k, w, y)\right)$.

$$
\prec_{Y}^{\prime j}=\prec_{Y}^{j}(j<k) \quad \prec_{Y}^{\prime j}=\left(\prec_{Y}^{j} \uparrow w\right)(j=k) \quad \prec_{Y}^{\prime j}=\emptyset(j>k) .
$$

Since $\varphi_{ \pm}$are $\Pi_{1}^{n+1}(\Gamma)$, by $\Pi_{1}^{n+1}(\Gamma)$-Red we have $H$ such that, for any $k, w, y$, (b) $\langle\langle k, w\rangle, y\rangle \in H \rightarrow \varphi_{+}(k, w, y)$ and $\langle\langle k, w\rangle, y\rangle \notin H \rightarrow \varphi_{-}(k, w, y)$.

We shall prove by induction on $\langle k, w\rangle$ along $\oplus_{k \in \omega} \prec_{H \uparrow k}^{k}$ that

$$
H_{\langle k, w\rangle}=\left\{y \mid \varphi\left(\langle k, w\rangle, y,(H)_{\left(\oplus_{k \in \omega} \prec_{H\lceil k}^{k}\right)\langle k, w\rangle}, \vec{x}, \vec{X}\right)\right\} .
$$

Thus the induction hypothesis is $\operatorname{Hier}[\varphi]\left(A,\left(\oplus_{j<k} \prec_{H\lceil j}^{j}\right) \oplus\left(\prec_{H\lceil k}^{k}\lceil w)\right)\right.$ where

$$
A=(H)_{\prec_{H}\langle k, w\rangle} \cup\left\{\left\langle\left\langle j, w^{\prime}\right\rangle, y\right\rangle \mid \varphi\left(\left\langle j, w^{\prime}\right\rangle, y, \emptyset, \vec{x}, \vec{X}\right) \& \neg\left(\left\langle j, w^{\prime}\right\rangle \prec_{H}\langle k, w\rangle\right)\right\},
$$

\footnotetext{
${ }^{11}(\forall X, k) \mathrm{WF}\left(\prec_{X \nmid k}^{k}\right)$ (uniform well-foundedness) might be too restricted. An alternative is $\operatorname{Prog} \mathrm{WF}[\prec](X) \equiv(\forall j<k) \mathrm{WF}\left(\prec_{X \mid j}^{j}\right) \wedge \operatorname{Hier}[\varphi]\left(X \mid k, \oplus_{j<k} \prec_{X \nmid j}^{j}\right) \rightarrow \mathrm{WF}\left(\prec_{X \mid k}^{k}\right)$ (progressive well-foundedness). If $(\forall X) \operatorname{Prog} W F[\prec](X)$ and $\operatorname{Hier}[\varphi]\left(H, \oplus_{k \in \omega} \prec_{H \nmid k}^{k}\right)$, then $\Delta_{0}^{n+1}(\mathrm{WF})$ induction implies $(\forall j<k) \mathrm{WF}\left(\prec_{H\lceil j}^{j}\right)$ and $\mathrm{WF}\left(\oplus_{k \in \omega} \prec_{H\lceil k}^{k}\right)$, which suffices for discussions below.

However, in $\mathcal{L}_{N}^{2}$, the induction is $\Pi_{1}^{1}$-Ind essentially and so beyond $\mathbf{A C A}_{0}$. The notion ProgWF itself seems impredicative and the avoidance of the difficulty by rules does not work.

If $\mathrm{WF}(-)$ is $\Delta_{0}^{n+1}$ (which is the case except in $\mathcal{L}_{N}^{2}$ as shown in $\S 8$ ), these two formulations are equivalent for $\Gamma=\Delta_{0}^{n+1}$, since $\operatorname{Prog} \mathrm{WF}[\prec](X)$ is just $(\forall k) \mathrm{WF}\left(\prec_{X \mid k}^{\prime k}\right)$ where:
}

$$
\prec_{X}^{\prime k}= \begin{cases}\prec_{X}^{k} & \text { if }(\forall j<k) \mathrm{WF}\left(\prec_{X \nmid j}^{j}\right) \wedge \operatorname{Hier}[\varphi]\left(X \mid k, \oplus_{j<k} \prec_{X \nmid j}^{j}\right) \\ \emptyset & \text { otherwise }\end{cases}
$$


with $\prec_{H}=\oplus_{k \in \omega} \prec_{H\lceil k}^{k}$, since, if $\neg\left(\left\langle j, w^{\prime}\right\rangle \prec_{H}\langle k, w\rangle\right)$ holds, $\left\langle j, w^{\prime}\right\rangle$ is minimal in the sense of $\left(\oplus_{j<k} \prec_{H\lceil j}^{j}\right) \oplus\left(\prec_{H \uparrow k}^{k}\lceil w)\right.$. Note that for $j \leq k$, by $A\lceil j=H\lceil j$,

$(\sharp) A^{k, w}=(A)_{\left(\oplus_{j \leq k} \prec_{\underline{A\lceil j}}^{j}\right)\langle k, w\rangle}=(A)_{\left(\oplus_{j \leq k} \prec_{\underline{H\lceil j}}^{j}\right)\langle k, w\rangle}=(H)_{\left(\oplus_{j \leq k} \prec_{H\lceil j}^{j}\right)\langle k, w\rangle}$.

Let $\pm\left(y \in H_{\langle k, w\rangle}\right)$, i.e., $\pm(\langle\langle k, w\rangle, y\rangle \in H)$. Then, by $(b)$, we have $\varphi_{ \pm}(k, w, y)$ and so $\pm \varphi\left(\langle k, w\rangle, y, A^{k, w}, \vec{x}, \vec{X}\right)$, which completes the induction by $(\sharp)$.

Corollary 24. Over $\Delta_{0}^{n+1}-\mathbf{C A}_{0}, \Pi_{e}^{n+1} \mathbf{- R e d}$ implies $\Delta_{e}^{n+1} \mathbf{-} \mathbf{T R} \mathbf{R}^{\sharp}$

REMARK 25. (i) This works well not only for 2-fold (internalized) autonomous progression with the preceding order $\omega$, but also for general multi-fold one.

(ii) In $\mathcal{L}_{N}^{2}$, since $\Pi_{e}^{1}$-Red and $\Delta_{0}^{1}$-TR are equivalent (see [15, Theorems V.5.1 and V.8.3]), $\Delta_{0}^{1}$-TR, $\Delta_{0}^{1}$-TR $\mathbf{R}^{\sharp}, \Delta_{e}^{1}$-TR and $\Delta_{e}^{1}-\mathbf{T R}^{\sharp}$ are all equivalent.

(iii) Thus relaxations $(\mathrm{E})$ and $\left(\mathrm{F}^{\prime}\right)$ from $\S 1$ (urged by multi-fold ones) do not change the limit of traditional predicativity, for $\Delta_{0}^{1}-\mathbf{T R} \mathbf{R}^{\sharp}$ is clearly embeddable in $\Delta_{0}^{1}-\mathbf{T} \mathbf{R}^{\sharp}$. This will turn out to be not the case for other kinds of predicativity.

Proposition 26. Over $\Delta_{0}^{n+1}-\mathbf{C A}_{0}, \Delta_{0}^{n+1}(\Gamma)$-FP implies $\Gamma$-TR $\mathbf{R}^{\sharp}$.

Proof. Following [2, Theorem 3.1]. For a given $\Gamma$-formula $\varphi\left(\langle k, w\rangle^{n}, y, Y\right)$, let $\varphi_{ \pm}$be the result of replacing as follows in $\varphi\left(\langle k, w\rangle^{n}, y,(Y)_{\left(\oplus_{k \in \omega} \prec_{Y \nmid k}^{k}\right)\langle k, w\rangle^{n}}\right)$ :

$$
\begin{array}{ccc} 
& \text { replace positive } t \in Y & \text { replace negative } t \in Y \\
\text { for } \varphi_{+} & \text {by }\langle 1, t\rangle^{n} \in F & \text { by }\langle 0, t\rangle^{n} \notin F \\
\text { for } \varphi_{-} & \text {by }\langle 0, t\rangle^{n} \notin F & \text { by }\langle 1, t\rangle^{n} \in F
\end{array}
$$

$F$ occurs only positively in $\varphi_{+}$and negatively in $\varphi_{-}$. For all $k, w, Y$ and $F$,

$$
\begin{gathered}
\left((F)_{1}\right)_{\left(\oplus_{j \in \omega} \prec_{Y \nmid j}^{j}\right)\langle k, w\rangle}=\left((F)_{0}{ }^{\mathbf{c}}\right)_{\left(\oplus_{j \in \omega} \prec_{Y \nmid j}^{j}\right)\langle k, w\rangle}=(Y)_{\left(\oplus_{j \in \omega} \prec_{Y \nmid j}^{j}\langle k, w\rangle\right.} \\
(\natural) \rightarrow \forall y\left[\varphi _ { + } ( k , w , y , F ) \leftrightarrow \varphi _ { - } ( k , w , y , F ) \leftrightarrow \varphi \left(\langle k, w\rangle, y,(Y)_{\left.\left.\left(\oplus_{k \in \omega} \prec_{Y \nmid k}^{k}\right)\langle k, w\rangle\right)\right],}\right.\right.
\end{gathered}
$$

where $^{\mathbf{c}}$ denotes complement, for the premise implies $(F)_{1}\left\lceil k=(F)_{0}{ }^{\mathbf{c}}\lceil k=Y \uparrow k\right.$.

Consider the positive $\Delta_{0}^{n+1}(\Gamma)$-operator form $\psi$ defined below:

$$
\psi(\langle i,\langle\langle k, w\rangle, y\rangle\rangle, Z) \equiv\left(i=1 \wedge \varphi_{+}(k, w, y, Z)\right) \vee\left(i=0 \wedge \neg \varphi_{-}(k, w, y, Z)\right) .
$$

$\Delta_{0}^{n+1}(\Gamma)$-FP yields $F$ such that $(\forall u)(u \in F \leftrightarrow \psi(u, F))$, i.e., for any $k, w, y$,

$(\diamond) \quad\langle\langle k, w\rangle, y\rangle \in(F)_{1} \leftrightarrow \varphi_{+}(k, w, y, F) ;\langle\langle k, w\rangle, y\rangle \in(F)_{0} \leftrightarrow \neg \varphi_{-}(k, w, y, F)$.

Letting $H=(F)_{1}$, we prove the following, by induction along $\oplus_{k \in \omega} \prec_{H \uparrow k}^{k}$ :

$$
\left((F)_{1}\right)_{\langle k, w\rangle}=\left((F)_{0}{ }^{\mathbf{c}}\right)_{\langle k, w\rangle}=(H)_{\langle k, w\rangle} .
$$

Now the induction hypothesis is the premise of $(\measuredangle)$ with $Y=H$, and so we have the conclusion of $(\natural)$ with $Y=H$, which implies, with $(\diamond)$, the statement. Now

$$
\langle\langle k, w\rangle, y\rangle \in H=(F)_{1} \leftrightarrow \varphi_{+}(k, w, y, F) \leftrightarrow \varphi\left(\langle k, w\rangle, y,(H)_{\left(\oplus_{k \in \omega} \prec_{H \nmid k}^{k}\right)\langle k, w\rangle}\right),
$$

holds for all $k, w, y$, which is the required equivalence.

REMARK 27. (i) Again, the proof works well also for general multi-fold one.

(ii) Avigad [2] provides a nice overview on the proof of the equivalence between $\Delta_{0}^{1}$-FP and $\Delta_{0}^{1}$-TR in $\mathcal{L}_{N}^{2}$, and thus this gives another proof of the equivalence between $\Delta_{0}^{1}$ - $\mathbf{T R}$ and $\Delta_{0}^{1}-\mathbf{T} \mathbf{R}^{\sharp}$ in second order number theory. 
(iii) Since the parameters play no role in the proof, $\widehat{I D}_{1}^{n+1}$ proves $\left(\Delta_{0}^{n+1}\right)^{-}-\mathbf{T R}^{\sharp}$ with the preceding order $\omega$ replaced by any provably well-founded relation.

$\S 8$. Well-foundedness and well-orderedness. So far, our uniform treatment works so well that we do not need to take care of the difference among $\mathcal{L}^{n+2}$ 's in the technical discussions. In this section, we are pointing out the basis which will cause all the differences among them in the later section.

The next lemma is what makes $\mathcal{L}_{N}^{2}$ be exceptional, since the well-foundedness of relations whose domain is (included in) $\omega$ is $\Pi_{1}^{1}$-complete in $\mathcal{L}_{N}^{2}$.

Lemma 28 (except $\mathcal{L}_{N}^{2}$ ). $\mathrm{WF}(R)$ is equivalent in $\Delta_{0}^{n+1}-\mathbf{C} \mathbf{A}_{0}$ to a $\Delta_{0}^{n+1}$-formula.

By Axiom (3), WF $(R)$ is equivalent to the non-existence of $R$-descending $\omega$ chain. Since an $\omega$-chain of $n+1$-th order objects can be coded by an $n+1$-th order object, $\mathrm{WF}(R)$ can be free from $n+2$-th order quantifiers, i.e., in $\Delta_{0}^{n+1}$.

Proof. We prove that $\neg \mathrm{WF}(R)$ is equivalent to the following:

$$
\left(\exists f^{n}\right)(\forall k \in \omega)\left[\left\langle(f)_{k},(f)_{k+1}\right\rangle^{n} \in R\right],
$$

where elements of $\omega$ are regarded as $n$-th order objects via the canonical injection and $(f)_{u}=\left\{v^{n-1} \mid\langle u, v\rangle^{n-1} \in^{n} f\right\}$ if $n \geq 1$ and where $(f)_{u}=f(u)$ in the usual sense of first order set theory, if $n=0$.

If $(\forall k \in \omega)\left[\left\langle(f)_{k},(f)_{k+1}\right\rangle^{n} \in R\right]$, then $X=\left\{x \mid(\exists k \in \omega)\left(x=(f)_{k}\right)\right\}$ satisfies

$$
(\forall x)\left[x \in X \rightarrow\left(\exists y \in(R)_{x}\right)(y \in X)\right]
$$

and so $X^{\prime}=\{x \mid x \notin X\}$ witnesses $\neg \mathrm{WF}(R)$ since $X$ is not empty.

Conversely, let $X^{\prime}$ witness $\neg \mathrm{WF}(R)$ and let $X=\left\{x \mid x \notin X^{\prime}\right\}$. Then we can take $g \in X$ and $(*)$ holds. Induction on $k \in \omega$ shows $\left(\exists ! h^{n}\right)\left(\exists f^{n}\right) \psi(h, f, k)$, where

$$
\begin{aligned}
\psi(h, f, k) \equiv(f)_{0}=g \wedge & (f)_{k}=h \wedge(\forall j<k)\left[\left\langle(f)_{j},(f)_{j+1}\right\rangle \in R \wedge\right. \\
& (\forall x)\left(\left\langle(f)_{j}, x\right\rangle \in R \rightarrow\left(x=(f)_{j+1} \vee\left\langle x,(f)_{j+1}\right\rangle \in W\right)\right]
\end{aligned}
$$

Then $f^{\prime}$ defined below satisfies $(\forall k \in \omega)\left[\left\langle\left(f^{\prime}\right)_{k},\left(f^{\prime}\right)_{k+1}\right\rangle^{n} \in R\right]$ :

$$
f^{\prime}=\left\{\begin{array}{ll}
\left\{\langle k, u\rangle^{n-1} \mid k \in \omega \wedge\left(\exists h^{n}, f^{n}\right)\left(\psi(h, f, k) \wedge u \in^{n} h\right\}\right. & \text { if } n \geq 1 \\
\left\{\langle k, u\rangle^{0} \mid k \in \omega \wedge\left(\exists h^{0}, f^{0}\right)(\psi(h, f, k) \wedge u=h)\right\} & \text { if } n=0
\end{array} .\right.
$$

Here, if $n=0$, we need the replacement scheme (and the axiom of infinity) to prove that $f^{\prime}$ exists as a first order object.

The need of replacement at the end explains why this lemma does not hold in $\mathcal{L}_{N}^{2}$. Actually, the replacement can substitute Axiom (3) of global well-order:

Lemma 29. $\mathrm{WF}(W)$ is equivalently $\Delta_{0}^{1}$ in NBG minus Axiom (3).

Proof. We prove the equivalence to $(\dagger)$ below. Clearly $\mathrm{WF}(W)$ implies $(\dagger)$.

$$
(\forall z)\left[(\forall x)\left(\left(\forall y \in(W)_{x}\right)(y \notin z) \rightarrow x \notin z\right) \rightarrow(\forall x)(x \notin z)\right] .
$$

Let $\neg \mathrm{WF}(W)$, say $x_{0} \notin X$ and $X$ is progressive along $W$. Here notice that the reflection principle for $\Delta_{0}^{1}$ formulae can be proved in the same way as in $\mathbf{Z F}$ (see $[11,7.4$ Theorem]), since all the axiom schemata are (especially the replacement 
is) now available for all $\Delta_{0}^{1}$ formulae with second order parameters. Thus, we have $a \ni x_{0}$ with $(a, a \cap X, a \cap W) \prec_{\Sigma_{2}}(V, X, W)$. Then $a \backslash X$ witnesses $\neg(\dagger) . \quad \dashv$

REMARK 30. Feferman (by private communication) raised up a question on the role of the foundation scheme in the elementarity of well-foundedness: does the lemma hold even in the absences of the foundation? Actually, the foundation scheme seems necessary to obtain the reflection principle, and, from some of the plausible notions of the universe of sets, the foundation scheme is not necessarily valid.

The answer is: again from the viewpoint of proof-theoretic strength (or consistency strength), foundation plays no role, because we can prove the equiconsistency between NBG, minus Axiom (3) of global well-order, augmented by some of the aforementioned additional axioms with and without the foundation scheme, by the relativization of the first and second order parts to, respectively,

$\mathrm{WF}=\{u \mid(\forall z)[(\forall x)((\forall y \in x)(y \notin z) \rightarrow x \notin z) \rightarrow(u \notin z)]\}$ and $\{X \mid X \subset \mathrm{WF}\}$.

Obviously, the relativization interprets NBG minus Axiom (3) of global wellorder and minus foundation, into itself, since elementary formulae are interpreted as elementary formulae. It also interprets the foundation scheme: for elementary $\varphi$, if $(\forall y \in x) \varphi^{\mathrm{WF}}(y) \rightarrow \varphi^{\mathrm{WF}}(x)$ holds for all $x \in \mathrm{WF}$, then, for any $u \in \mathrm{WF}$, $z=\left\{y \in \operatorname{trcl}(u \cup\{u\}) \mid \neg \varphi^{\mathrm{WF}}(y)\right\}$, yielded by the separation scheme, satisfies $(\forall x)((\forall y \in x)(y \notin z) \rightarrow x \notin z)$ and so, by $u \in \mathrm{WF}, u \notin z$, i.e., $\varphi^{\mathrm{WF}}(u)$.

Let us show, for example, that $\Delta_{0}^{1}$-FP is interpreted by this relativization into the systems with $\Delta_{0}^{1}$-FP: if $X$ occurs only positively in $\varphi(y, X)$, then also in $\varphi^{\mathrm{WF}}(y, X \cap \mathrm{WF})$ and hence $\Delta_{0}^{1}$-FP yields $F$ such that $(\forall y)[y \in F \leftrightarrow$ $\left.\varphi^{\mathrm{WF}}(y, F \cap \mathrm{WF})\right]$, i.e., $\mathrm{WF} \models(\forall y)\left[y \in F^{\prime} \leftrightarrow \varphi\left(y, F^{\prime}\right)\right]$, for $F^{\prime}=F \cap \mathrm{WF}$.

One might ask if the right $\mathcal{L}_{S}^{2}$-analogue of well-foundedness is the different notion, the non-existence of descending Ord-chains (called weak-well-foundedness in [4]). Though this new notion plays some roles played in $\mathcal{L}_{N}^{2}$ by well-foundedness, this does not allow definitions by recursion. (While Flumini [4] invented weak induction schema along weak-well-founded relations, an analogue for recursion is hopeless as shown by Flumini and Sato [5].) Thus it seems impossible to argue against the privileged status of the notion of well-foundedness, even in $\mathcal{L}_{S}^{2}$ (and in $\mathcal{L}^{n+2}$ for $n \geq 1$ ).

Let us close this section, by pointing out that a similar phenomenon is known in higher order recursion theory: the theory of type- $n$ functional for $n \geq 3$ is quite different from that of type-2 functional, as explained, e.g., in Chapter VII "Recursion in Type-3 Functional" from Hinman [8], where he wrote:

...this chapter is not the second in an infinite sequence. Although there are several important differences between the theories of recursion relative to functionals of types 2 and 3, most of the theory of recursion relative to functionals of types greater than 3 can be obtained from type-3 theory with essentially only notational changes. $\quad[8$, p.343]

The basis for this discrepancy is that the property of well-foundedness for type-2 relations is $\Delta_{1}^{2}$ (in fact $\Delta_{(\omega)}^{1}$, Lemma VI.7.11), whereas wellfoundedness for type- 1 relations is $\Pi_{1}^{1}$ but not $\Delta_{1}^{1}$.

$[8, \mathrm{p} .355]$ 
§9. Main result. In this section, we prove the main result: "external" 2fold autonomous progression proves the consistency of boldface "internalized" single autonomous progression $\Delta_{0}^{n+1}$ - TR with several axiomatic schemata, if well-foundedness is $\Delta_{0}^{n+1}$. Thus the former system is proof-theoretically strictly stronger than the latter and that the latter does not imply $\Delta_{1}^{n+1}$-CA.

The key notion is coded lower order parts sharing (LOPS, for short) model, which generalizes the notion of coded $\omega$-model in $\mathcal{L}_{N}^{2}$ (see [15, VII.2]).

Definition 31. A coded LOPS model is a $n+2$-th order object $M$, viewed as encoding the $\mathcal{L}^{n+2}$-structure whose $k$-th order part consists of

$$
\left\{x^{k-1} \mid x=x\right\} \text { for } 0<k \leq n+1 ; \quad\left\{(M)_{x} \mid x \in \mathcal{P}^{n}\right\} \text { for } k=n+2 .
$$

REMARK 32. " $M \models \varphi$ " is $\Delta_{0}^{n+1}$ for any $\mathcal{L}^{n+2}$-formula $\varphi$, since $M \models(Q X) \varphi(X)$ is $\left(Q x^{n}\right)\left(M \models \varphi\left((M)_{x}\right)\right)$. Thus, any LOPS-model satisfies (i) in $\mathcal{L}_{N}^{n+2}$, the full induction $\left(\mathcal{L}_{N}^{n+2}\right.$-Ind); (ii) in $\mathcal{L}_{S}^{n+2}$, the full induction, foundation, separation and replacement $\left(\mathcal{L}_{S}^{n+2}\right.$-Ind, -Found, -sep, -repl); (iii) in the both, $k+1$-th order $\mathcal{L}^{n+2}$ comprehension for $k \leq n+1$ (i.e., Axiom (2) extended to all formulae).

TheOREM 33 (except $\left.\mathcal{L}_{N}^{2}\right) . \Delta_{0}^{n+1}-\mathbf{C A}_{0}+\left(\Delta_{0}^{n+1}\right)^{-}-\mathbf{T R} \mathbf{R}^{\sharp}$ proves the existence of a coded LOPS-model of $\Delta_{0}^{n+1}-\mathbf{C A}_{0}+\Delta_{0}^{n+1}-\mathbf{T R}$.

We will define LOPS models $M_{k}$ 's recursively as follows. Given $M_{k}$, let $\sqsubset_{M_{k}}^{k}$ be the disjoint union of all well-founded relations in $M_{k}$, which are, by absoluteness, "really" well-founded. Let $M_{k+1}$ consist of all the sets obtainable by $\Delta_{0}^{n+1}$ transfinite recursion along $\sqsubset_{M_{k}}^{k} . M_{k+1}$ is definable by a single transfinite recursion with the universal $\Sigma_{2}^{n}$-formula $\Upsilon_{2}^{n}$, induced by $\Upsilon_{1}^{n} . \quad M=\bigcup_{k<\omega} M_{k}$ is yielded by $\Delta_{0}^{n+1} \mathbf{-} \mathbf{T R R} \mathbf{R}^{\sharp}$ along some $\prec_{X}^{k}$ (such that $\prec_{M \uparrow k}^{k+1}=\sqsubset_{M_{k}}^{k}$ ) and is the required model.

Proof. Let $\Upsilon_{2}^{n}(y, Y, Z)$ be the universal $\Sigma_{2}^{n}$-formula with two $n+2$-th order variables. By $\Delta_{0}^{n+1} \mathbf{T R R}^{\sharp}$ we have $H$ with $\operatorname{Hier}[\psi]\left(H, \oplus_{k \in \omega} \prec_{H \uparrow k}^{k}\right)$, where:

$\psi(\langle k, x\rangle,\langle c, a\rangle, X) \leftrightarrow \Upsilon_{2}^{n}(c,\langle x, a\rangle,\{\langle y, b\rangle \mid\langle\langle k, y\rangle,\langle c, b\rangle\rangle \in X\}, X\lceil k) ;$

$M_{k}(X)=\left\{\langle\langle 2 j, c\rangle,\langle x, a\rangle\rangle,\langle\langle 2 j+1,\langle c, x\rangle\rangle, a\rangle \mid j<k \wedge a \in\left((X)_{\langle j, x\rangle}\right)_{c}\right\} ;$

$x \prec_{X}^{k} y \leftrightarrow\left(\exists a, x^{\prime}, y^{\prime}\right)\left[\mathrm{WF}\left(\left(M_{k}(X)\right)_{a}\right) \wedge x=\left\langle a, x^{\prime}\right\rangle \wedge y=\left\langle a, y^{\prime}\right\rangle \wedge\left\langle x^{\prime}, y^{\prime}\right\rangle \in\left(M_{k}(X)\right)_{a}\right]$.

We can see $M_{k}(H)=M_{k}\left(H\lceil k)\right.$, which we denote by $M_{k}$, and $M_{k}$ 's are increasing as a sequence of LOPS models. Let $M=\bigcup_{k \in \omega} M_{k}$. Hier $[\psi]\left(H, \oplus_{k \in \omega} \prec_{H\lceil k}^{k}\right)$ is:

$$
\begin{aligned}
(H)_{\langle k, x\rangle} & =\left\{\langle c, a\rangle \mid \Upsilon_{2}^{n}\left(c,\langle x, a\rangle,\left\{\langle y, b\rangle \mid\langle\langle k, y\rangle,\langle c, b\rangle\rangle \in H_{\left(\oplus_{j \in \omega} \prec_{H \nmid j}^{j}\right)\langle k, x\rangle}\right\}, H\lceil k)\right\}\right. \\
(\mathbf{W}) & =\left\{\langle c, a\rangle \mid \Upsilon_{2}^{n}\left(c,\langle x, a\rangle,\left\{\langle y, b\rangle \mid\langle c, b\rangle \in(H)_{\langle k, y\rangle}, y \prec_{H\lceil k}^{k} x\right\}, H\lceil k)\right\} .\right.
\end{aligned}
$$

For a $\Sigma_{2}^{n}$-formula $\varphi$ with parameters from $M_{k}$, since parameters are $\Sigma_{1}^{n}$-definable from $H\left\lceil k\right.$, there is $c$ such that $(\forall x, a)\left(\varphi(x, a, Y) \leftrightarrow \Upsilon_{2}^{n}(c,\langle x, a\rangle, Y, H\lceil k))\right.$.

Let us first consider the case where $Y$ does not occur in $\varphi$. ( implies

$$
\left(M_{k+1}\right)_{\langle 2 k+1,\langle c, x\rangle\rangle}=\left((H)_{\langle k, x\rangle}\right)_{c}=\left\{a \mid \Upsilon_{2}^{n}(c,\langle x, a\rangle,-, H\lceil k)\}=\{a \mid \varphi(x, a)\} .\right.
$$

Thus, by the absoluteness of $\Delta_{0}^{n+1}$-formulae, $M \models \Sigma_{2}^{n}$-CA and so $M \models \Delta_{0}^{n+1}-\mathbf{C A} \mathbf{A}_{0}$. 
For $G=\left(M_{k+1}\right)_{\langle 2 k, c\rangle}=\left\{\langle x, a\rangle \mid a \in\left((H)_{\langle k, x\rangle}\right)_{c}\right\}$ in $M_{k+1}$, (W) implies $(G)_{x}=\left\{a \mid \varphi\left(x, a,\left\{\langle y, b\rangle \mid b \in\left((H)_{\langle k, y\rangle}\right)_{c}, y \prec_{H\lceil k}^{k} x\right\}\right)\right\}=\left\{a \mid \varphi\left(x, a,(G)_{\prec_{H\lceil k}^{k} x}\right)\right\}$,

i.e., $\operatorname{Hier}[\varphi]\left(\prec_{H\lceil k}^{k}, G\right)$. Then $M \models\left(\exists G^{\prime}\right) \operatorname{Hier}[\varphi]\left(W, G^{\prime}\right)$ for any $W$ with $\operatorname{WF}(W)$ from $M_{k}$, since $W$ is included in $\prec_{H \uparrow k}^{k}$. Thus, by Footnote $5, M \models \Delta_{0}^{n+1}$-TR. $\dashv$

If iterated comprehension (even only up to $\omega$ ) for $\Delta_{0}^{n+1}$-formula containing $M$ as a parameter is available, we can define the truth predicate relative to $M$, by which we can prove the consistency of the theory satisfied by $M$. Thus,

Corollary 34 (except $\mathcal{L}_{N}^{2}$ ). For schemata '-Sch' mentioned in Remark 32, $\Delta_{0}^{n+1}-\mathbf{C A}_{0}+\Delta_{0}^{n+1} \mathbf{-} \mathbf{T} \mathbf{R}^{\sharp} \vdash \operatorname{Con}\left(\Delta_{0}^{n+1}-\mathbf{C A}_{0}+\Delta_{0}^{n+1} \mathbf{- T R}+\mathcal{L}^{n+2} \mathbf{- S c h}\right)$.

REMARK 35. Since we need transfinite recursion to define the truth predicate of $M$ after $M$ is defined, $\left(\Delta_{0}^{n+1}\right)^{-}$- $\mathbf{T R R} \mathbf{R}^{\sharp}$ does not seem to prove the consistency. However, if we replace the "preceding order" $\omega$ in $\left(\Delta_{0}^{n+1}\right)^{-}-\mathbf{T R} \mathbf{R}^{\sharp}$ by $\omega+1$ (i.e., if we allow $\omega+1$-th iteration of non-dependent transfinite recursion; see also $\Gamma$ - $\mathbf{T} \mathbf{R}^{2}$ in $\left.\S 10\right)$, then the consistency can be proved. As mentioned in Remark 27 (iii), $\widehat{I D}_{1}^{n+1}$ implies $\left(\Delta_{0}^{n+1}\right)^{-}-\mathbf{T R R} \mathbf{R}^{\sharp}$ with preceding order $\omega+1$. Thus, we have

$$
\widehat{I D}_{1}^{n+1} \vdash \operatorname{Con}\left(\Delta_{0}^{n+1}-\mathbf{C A}_{0}+\Delta_{0}^{n+1} \mathbf{- T R}+\mathcal{L}^{n+2} \mathbf{- S c h}\right) .
$$

Note that $\widehat{I D}_{1}^{n+1}$ can be interpreted in $\Delta_{0}^{n+1} \mathbf{- C A} \mathbf{A}_{0}+\Sigma_{1}^{n+1} \mathbf{- C o l l}+\Delta_{0}^{n+1}\left(\Sigma_{1}^{n+1}\right)-\mathbf{S c h}$ by diagonalizing $\Upsilon_{1}^{n+1}$ in the same way as in $\mathcal{L}_{N}^{2}$ (see [1]).

REMARK 36. The theorem can be generalized for $\Gamma$ - $\mathbf{T R} \sharp$ and $\Gamma$-TR if (i) $\Gamma$ includes all those formulae elementary in well-foundedness; (ii) $\Gamma$-formulae is absolute for the LOPS models. In $\mathcal{L}_{N}^{2}$, the condition (i) prevents us from having the result for $\Delta_{0}^{1}$. However, for $\Pi_{1}^{1}$ in $\mathcal{L}_{N}^{2}$, because of Kleene's basis theorem [15, VII.1.8], the LOPS model in the proof is a $\beta$-model and so the two conditions are satisfied. Thus $\mathbf{A} \mathbf{C A} \mathbf{A}_{0}+\Pi_{1}^{1}-\mathbf{T} \mathbf{R}^{\sharp} \vdash \operatorname{Con}\left(\mathbf{A C A} \mathbf{A}_{0}+\Pi_{1}^{1} \mathbf{- T R}+\mathcal{L}^{2} \mathbf{- S c h}\right)$ in $\mathcal{L}_{N}^{2}$.

Corollary $37\left(\operatorname{except} \mathcal{L}_{N}^{2}\right) . \Delta_{0}^{n+1}-\mathbf{C A}_{0}+\Delta_{0}^{n+1}-\mathbf{T R}+\mathcal{L}^{n+2} \mathbf{- S c h} \forall \Delta_{1}^{n+1} \mathbf{- C A}$.

Proof. Let $T$ be the system. If $T \vdash \Delta_{1}^{n+1}-\mathbf{C A}$, since $T$ includes $\Sigma_{1}^{n+1}$-Ind, by Lemma 21, $T \vdash \Delta_{0}^{n+1}-\mathbf{T R}^{\sharp}$ and, by the theorem, $T \vdash \operatorname{Con}(T)$.

By Lemma 15, $\Delta_{0}^{n+1}-\mathbf{C A}_{0}+\Delta_{0}^{n+1} \mathbf{- T R}+\mathcal{L}^{n+2}$-Sch $\forall \Sigma_{1}^{n+1}$-Coll follows, contrasting with the known result $\mathbf{A C A}_{0}+\Delta_{0}^{1}$-TR $\vdash \Sigma_{1}^{1}$-Coll (e.g., [15, V.8.3]).

REMARK 38. Proof-theoretically, however, $\Sigma_{1}^{n+1}$-Coll (nor $\Delta_{1}^{n+1}$-CA) does not affect $\Delta_{0}^{n+1}-\mathbf{C A}_{0}+\Delta_{0}^{n+1}$-TR. More generally, for a $\Delta_{0}^{n+1}$-formula $\Psi(X, Y)$, we can establish the $\Pi_{2}^{n+1}$-conservation of adding $\Sigma_{1}^{n+1}$-Coll to $\Delta_{0}^{n+1}-\mathbf{C A}_{0}+$ $(\forall X)(\exists Y) \Psi(X, Y)$, when $\Delta_{0}^{n+1}-\mathbf{C A}_{0}+\forall X \exists Y \Psi(X, Y) \vdash \forall X \exists Y \forall z \Psi\left((X)_{z},(Y)_{z}\right)$, in the same way as the proof of $\left[15\right.$, IX.4.4] with "TJ $\left(A_{n}\right)=A_{n+1}$ " replaced by "TJ $\left(A_{2 n}\right)=A_{2 n+1} \wedge(\forall z) \Psi\left(\left(A_{2 n+1}\right)_{z},\left(A_{2 n+2}\right)_{z}\right) \wedge \forall x \exists y\left(A_{n}\right)_{x}=\left(A_{n+1}\right)_{y}$ ". Note that the proof of our main theorem shows how to obtain such $\Psi$ for $\Delta_{0}^{n+1}$-TR. 
§10. Further problems. Since dependent transfinite recursion is a new kind of axiom scheme, there are many questions open. For example,

- Since the proof of Theorem 33 does not work for classes other than $\Delta_{0}^{n+1}$ (except $\Pi_{1}^{1}$ in $\mathcal{L}_{N}^{2}$ as discussed in Remark 36), we have no idea if $\Pi_{k+1}^{n+1}$-TR $\mathbf{R}^{\sharp}$ is strictly stronger than $\Pi_{k+1}^{n+1}$-TR. This problem survives in $\mathcal{L}_{N}^{2}$ for $n \geq 2$, while we have Propositions 23 and 26.

- While Corollaries 24 and 22 (a) position $\Delta_{e}^{n+1}$-TR (or with $\sharp$ ) between $\Pi_{1}^{n+1}$-Red $+\Sigma_{1}^{n+1}$-Coll and $\Delta_{0}^{n+1}-\mathbf{T R} \mathbf{R}^{\sharp}$ (in the presence of sufficient induction), is it properly between? Or what are the relations with $\Delta_{0}^{n+1} \mathbf{- F P}$ or with $\Pi_{1}^{n+1}$-Red (alone)?

- Autonomous progression can be considered not only for comprehension but also for other constructions. Among them, Strahm [16] considered autonomous progression of fixed-point principle for positive elementary operators and its "internalized version" FTR. It is natural to ask if $\mathbf{F T R}^{\sharp}$ is stronger than FTR both in number and set theories, and the same question in general $\mathcal{L}^{n+2}$ for positive $\Pi_{k}^{n+1}$ operators.

However, the most important problem seems to be: to capture (or to give a formulation to) the whole scope of dependent transfinite recursion (or, multifold autonomous progression). As remarked several times, in the definition of $\Gamma$ - $\mathbf{T R} \mathbf{R}^{\sharp}, n$ 's in $\prec_{Y}^{n}$ can be replaced by any ordinal, or $\omega$ in $\oplus_{n \in \omega} \prec_{Y\lceil n}^{n}$ can be replaced by any well-founded relation. This can be formalized as follows.

$\Gamma-\mathbf{T R}^{2}: \operatorname{WF}\left(\prec^{(1)}\right) \wedge(\forall w, X) \mathrm{WF}\left(\prec_{X \mid w}^{(0), w}\right) \rightarrow(\exists H) \operatorname{Hier}[\varphi]\left(H, \oplus_{w \in \operatorname{fd}\left(\prec^{(1)}\right)} \prec_{H \uparrow w}^{(0), w}\right)$, for any $\Gamma$-formulae $\prec^{(1)}$ and $\prec_{Y}^{(0), w}$ and for any $\Gamma$-formula $\varphi$.

$\prec^{(1)}$ was called "preceding order" in Remarks 25, 27 and 35. We can further generalize it by allowing $\prec^{(1)}$ to depend on $H$. Iterating this generalization,

$$
\Gamma-\mathbf{T R}^{k+1}:(\forall \vec{w}, Y) \bigwedge_{j \leq k} \mathrm{WF}\left(\prec_{Y \uparrow\left\langle w_{k}, \cdots, w_{j+1}\right\rangle}^{(j), w_{k}, \cdots, w_{j+1}}\right) \rightarrow(\exists H) \operatorname{Hier}[\varphi]\left(H, \Pi_{j \leq k} \prec_{H}^{(j)}\right)
$$

for any $\Gamma$-formulae $\varphi$ and $\prec_{Y}^{(j), w^{k}, \cdots, w^{j+1}}$ (for $j<k$ ), where $\vec{w}\left(\Pi_{j \leq k} \prec_{H}^{(j)}\right) \vec{v}$ is defined as $\left(w_{k} \prec^{(k)} v_{k}\right) \vee \cdots \vee\left(w_{k}=v_{k} \wedge \cdots \wedge w_{1}=v_{1} \wedge w^{0} \prec_{H \uparrow\left\langle w_{n}, \cdots, w_{1}\right\rangle}^{(0), w_{k}, \cdots, w_{1}} v_{0}\right)$.

Furthermore we can define $\Gamma$-TR ${ }^{\omega}$, by considering $\omega$-sequences $f$ such that, for all but finite $k \in \omega, f(k)$ is the minimum in $\prec_{X}^{f \uparrow(\omega \backslash(k+1))}$ (like Veblen hierarchy), and we can replace this $\omega$ by any well-order, which, again, depends on the intermediate stage of resulting hierarchy $H$, and so on. Notice that all these extensions are implied both by $\Pi_{1}^{n+1}(\Gamma)$-Red and $\Delta_{0}^{n+1}(\Gamma)-\mathbf{F P}$, since the proofs of Propositions 23 and 26 survive as reminded before whereas, in order to let Corollary 22 survive, we need to replace $\Sigma_{e}^{n+1}$-Ind by $\Sigma_{e}^{n+1}$-TI.

How can we formalize all these extensions in one schema? Such a scheme should be called the full dependent transfinite recursion and denoted by -DTR. To capture the limit of relative predicativity, we need such one, if we agree that multi-fold autonomous progressions are also accepted in relative predicativity. However, for this we need an invention as breakthrough as the extraction of wellorderedness from "transfiniteness" or processes going beyond length $\omega$ (although the invention has not been made properly yet, as discussed in $\S 6$.) 
§11. Conclusions. We have seen that the traditional predicativity, namely "predicativity given $\omega$ ", is quite different from "predicativity given the totality of all real numbers", from "predicativity given the totality of all functions" and from "predicativity given the universe of sets", in the following sense: the relations among the central notions, i.e., single and multi-fold autonomous progressions of $\Delta_{0}^{n+1}$ and $\Delta_{e}^{n+1}$ comprehension, are completely different, and the predicative reducibility of some axioms, i.e., $\Pi_{1}^{n+1}$ reduction and $\Delta_{0}^{n+1}$ fixed point, holds to traditional predicativity but seems to fail to the other kinds of predicativity: the dependent autonomous progression is bounded from above by parameterfree version of $\Pi_{1}^{n+1}$ reduction (which we do not officially define) and that of $\Delta_{0}^{n+1}$ fixed point (namely $\widehat{I D}_{1}^{n+1}$ ), which must be strictly weaker than the usual parameter-allowed versions.

Differences are summarized in Table 1 , where the base theory is $\Delta_{0}^{n+1}-\mathbf{C A} \mathbf{A}_{0}+$ $\Gamma$-TI for any $\Gamma \subset \mathcal{L}^{n+2}$, except that $\Gamma \supset \Sigma_{e}^{n+1}$ when $\Delta_{e}^{n+1}-\mathbf{T R}^{(\sharp)}$ is concerned.

\begin{tabular}{|c|c|}
\hline in $\mathcal{L}_{N}^{2}$ & in $\mathcal{L}_{N}^{n+2}(n \geq 1)$ and $\mathcal{L}_{S}^{n+2}(n \geq 0)$ \\
\hline$\Delta_{e}^{1} \mathbf{- T R}^{(\sharp)} \leftrightarrow \Delta_{0}^{1} \mathbf{- T R}^{\sharp} \leftrightarrow \Delta_{0}^{1}$-TR & $\Delta_{e}^{n+1} \mathbf{-} \mathbf{T R}^{(\sharp)}, \Delta_{0}^{n+1} \mathbf{-} \mathbf{T R} \mathbf{R}^{\sharp} \rightarrow \operatorname{Con}\left(\Delta_{0}^{n+1} \mathbf{- T R}\right)$ \\
\hline$\Pi_{1}^{1}$-Red $\leftrightarrow \Delta_{0}^{1}$-FP $\leftrightarrow \Delta_{0}^{1}$-TR & $\Pi_{1}^{n+1}$-Red, $\Delta_{0}^{n+1} \mathbf{- F P} \rightarrow \operatorname{Con}\left(\Delta_{0}^{n+1}\right.$-TR $)$ \\
\hline$\Delta_{0}^{1}-\mathbf{T R} \rightarrow \operatorname{Con}\left(\widehat{I D}_{1}^{1}\right)$ & $\widehat{I D}_{1}^{n+1} \rightarrow \operatorname{Con}\left(\Delta_{0}^{n+1} \mathbf{- T R}\right)$ \\
\hline$\Delta_{0}^{1}$-TR $\rightarrow \Delta_{1}^{1}$-CA, $\Sigma_{1}^{1}$-Coll & $\Delta_{0}^{n+1}$-TR $\nrightarrow \rightarrow \Delta_{1}^{n+1}$-CA,$\Sigma_{1}^{n+1}$-Coll \\
\hline
\end{tabular}
TABLE 1. the difference between $\mathcal{L}_{N}^{2}$ and the other $\mathcal{L}^{n+2}$ 's

These results suggest a new trend of research: to answer the following question.

Among the known results in $\mathcal{L}_{N}^{2}$, which hold in general $\mathcal{L}^{n+2}$ and which are specific to $\mathcal{L}_{N}^{2}$ (i.e., do not hold in $\mathcal{L}^{n+2}$ other than $\mathcal{L}_{N}^{2}$ ).

More finely, which holds in which instance of $\mathcal{L}^{n+2}$ ? Indeed, this trend, restricted to $\mathcal{L}_{S}^{2}$, has already mentioned in Krähenbühl [10], Fujimoto [7] and Flumini [4], and actually been executed in several papers (e.g., Jäger and Krähenbühl [9], as well as [10], [7] and [4]). Though one might think that these results are straightforward generalizations of results known in $\mathcal{L}_{N}^{2}$, our results in the present paper show that the trend of research cannot be trivial.

Related to this trend, one interesting question is: what is the right analogue of ACA or of general "naught-less" $\mathcal{L}_{N}^{2}$-theories? Jäger and Krähenbühl [9] employ the view that "naught-less" in $\mathcal{L}_{N}^{2}$ corresponds to "adding full foundation" in $\mathcal{L}_{S}^{2}$ and show that this view works very well particularly in the context of infinitary proof systems. On the other hand, Fujimoto [7, Remark 1] claims that it should correspond to "adding the foundation, separation and replacement schemata for the full language", which works well for, e.g., the embedding of $\widehat{I D}_{1}^{n+1}$ mentioned at the end of Remark 35. The difference occurs clearly in the case of $\Sigma_{1}^{1}$-Coll:

(I) by Remark $38, \mathbf{N B G}+\Sigma_{1}^{1}$-Coll is $\Pi_{2}^{1}$-conservative over $\mathbf{N B G}$;

(II) [10] shows that $\mathbf{N B G}+\Sigma_{1}^{1}$-Coll $+\mathcal{L}_{S}^{2}$-Found is $\Pi_{2}^{1}$-conservative over what they call $\mathbf{N B G} \mathbf{E}_{<0}$, whose strength is between $\mathbf{N B G}$ and $\mathbf{N B G}+\Delta_{0}^{1}-\mathbf{T R}$;

(III) Remark 35 (and the final remark of Section 6 in [7]) asserts NBG + $\Sigma_{1}^{1}$-Coll $+\mathcal{L}_{S}^{2} \mathbf{- S c h}$ is strictly stronger than $\mathbf{N B G}_{0}+\Delta_{0}^{1}-\mathbf{T R}$.

It seems plausible that all these results hold in general $\mathcal{L}^{n+2}$, except $\mathcal{L}_{N}^{2}$. 
Finally, the author would like to emphasize that this new trend of research can be seen as a continuation of his previous researches [12] and [13] on the comparison among second order frameworks, since $\mathcal{L}^{n+2}$ 's can be seen as second order frameworks by considering objects of less than $n+2$-th order as first order objects and objects of $n+2$-th order as second order ones.

Acknowledgments. The author greatly appreciates very stimulating discussions with Solomon Feferman and Kentaro Fujimoto, an ex-student of Feferman's. He would like to appreciate the hospitality of members of Logic and Theory Group at Institut für Informatik und angewandte Mathematik, Universität Bern, during his stay at which he obtained most results of this article, especially discussions with Gerhard Jäger, Thomas Strahm and Dandolo Flumini. This article has been prepared afterward, during the author's employment at Institut für Informatik und angewandte Mathematik, Universität Bern.

\section{REFERENCES}

[1] P. ACZEL, The strength of Martin-Lö's type theory with one universe, Technical report, Department of Philosophy, University of Helsinki, 1977.

[2] J. AvigAD, On the relationship between $A T R_{0}$ and $\widehat{I D}_{<\omega}$, this JouRnAL, vol. 61 (1996), no. 3, pp. $768-779$.

[3] S. FEFERMAn, Predicativity, The Oxford handbook of the philosophy of mathematics and logic (S. Shapiro, editor), Oxford University Press, 2005, pp. 590-624.

[4] D. Flumini, Weak well orders, Ph.D. thesis, Universität Bern, 2013.

[5] D. Flumini and K. SAto, From hierarchies to well foundedness, Archive for Mathematical Logic, (2013), accepted for publication, www.iam.unibe.ch/ltgpub/2014/fs14.pdf.

[6] H. Friedman, Higher set theory and mathematical practice, Annals of Mathematical Logic, vol. 2 (1971), no. 3, pp. 325-357.

[7] K. Fujimoto, Classes and truths in set theory, Annals of Pure and Applied Logic, vol. 163 (2012), no. 11, pp. 1484-1523.

[8] P. Hinman, Recursion-theoretic hierarchies, Springer-Verlag, 1970.

[9] G. JÄGER and J. KRÄHENBüHL, $\Sigma_{1}^{1}$ choice in a theory of sets and classes, Ways of proof theory (R. Schindler, editor), Ontos Verlag, 2010, pp. 283-314.

[10] J. KRÄHENBüHL, On the relationship between choice schemes and iterated class comprehension in set theory, Ph.D. thesis, Universität Bern, 2011.

[11] K. Kunen, Set theory: An introduction to consistency proofs, North Holland, 1983.

[12] K. SATo, The strength of extensionality I: Weak weak set theories with infinity, Annals of Pure and Applied Logic, vol. 157 (2009), no. 2-3, pp. 234-268.

[13] - The strength of extensionality II: Weak weak set theories without infinity, Annals of Pure and Applied Logic, vol. 162 (2011), no. 8, pp. 579-646.

[14] , The strength of extensionality III: Weak weak set theories with the higher infinite, in preparation, $20 \infty$.

[15] S. SIMPSON, Subsystems of second order arithmetic, Springer-Verlag, 1999.

[16] T. Strahm, Autonomous fixed point progressions and fixed point transfinite recursion, Logic colloquium 98 (S. Buss, P. Hajek, and P. Pudlak, editors), Association of Symbolic Logic, 2000, pp. 449-464.

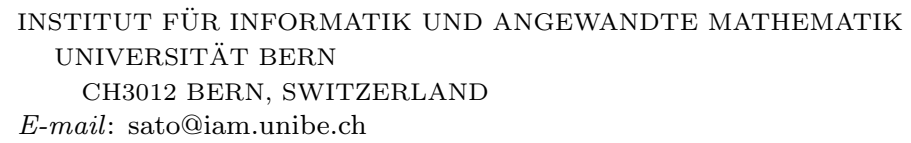

